\title{
Electric dipole moment constraints on CP-violating heavy-quark Yukawas at next-to-leading order
}

\author{
Joachim Brod ${ }^{a, b}$ and Emmanuel Stamou ${ }^{b, c, d}$ \\ ${ }^{a}$ Department of Physics, University of Cincinnati, \\ 345 Clifton Ct, Cincinnati, OH 45221, U.S.A. \\ ${ }^{b}$ Fakultät für Physik, TU Dortmund, \\ Otto-Hahn-Straße 4, Dortmund 44227, Germany \\ ${ }^{c}$ Theoretical Particle Physics Laboratory (LPTP), Institute of Physics, EPFL, \\ Route de la Sorge, 1015 Lausanne, Switzerland \\ ${ }^{d}$ Enrico Fermi Institute, University of Chicago, \\ 5640 S Ellis Ave, Chicago, IL 60637, U.S.A. \\ E-mail: joachim.brod@uc.edu, emmanuel.stamou@tu-dortmund.de
}

ABSTRACT: Electric dipole moments are sensitive probes of new phases in the Higgs Yukawa couplings. We calculate the complete two-loop QCD anomalous dimension matrix for the mixing of CP-odd scalar and tensor operators and apply our results for a phenomenological study of $\mathrm{CP}$ violation in the bottom and charm Yukawa couplings. We find large shifts of the induced Wilson coefficients at next-to-leading-logarithmic order. Using the experimental bound on the electric dipole moments of the neutron and mercury, we update the constraints on CP-violating phases in the bottom and charm quark Yukawas.

Keywords: Beyond Standard Model, CP violation, Higgs Physics, Quark Masses and SM Parameters

ArXIV EPRINT: 1810.12303 


\section{Contents}

1 Introduction 1

2 Effective theory below the weak scale $\quad 2$

3 Renormalisation group evolution 5

$\begin{array}{ll}3.1 & \text { Initial conditions at the weak scale }\end{array}$

$\begin{array}{ll}3.2 & \text { Calculation of the anomalous dimensions }\end{array}$

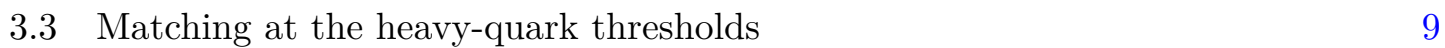

4 Numerics 11

4.1 Wilson coefficients 11

$\begin{array}{lll}4.2 & \text { Bounds on CP phases from hadronic EDMs } & 14\end{array}$

5 Conclusions 18

$\begin{array}{ll}\text { A Unphysical operators } & 19\end{array}$

A.1 E.o.m.-vanishing operators 20

A.2 Evanescent operators 21

A.3 Operators related to the infrared rearrangement 24

B Renormalisation constants $\quad 24$

$\begin{array}{ll}\text { C The one-loop anomalous dimensions } & 27\end{array}$

$\begin{array}{ll}\text { D Change of renormalisation scheme } & 28\end{array}$

$\begin{array}{ll}\text { E Expanding the renormalisation group } & 30\end{array}$

\section{Introduction}

With the discovery of the Higgs boson in 2012, the precise determination of its couplings to all other standard model (SM) particles became a primary goal of particle physics. Of special interest are quark-Yukawa couplings. In the SM, they are real and proportional to the quark masses; any deviation from this relation would indicate physics beyond the SM. Such new contributions to Yukawa couplings are often unavoidable in extensions of the SM that predict new particles at the LHC.

Non-SM Yukawa interactions may in fact be related to the dynamics underlying baryogenesis. For instance, new sources of $\mathrm{CP}$ violation are required for electroweak baryogenesis (see ref. [1] for a review). Various models of electroweak baryogenesis require a sizeable phase in the top Yukawa. Naively, such a phase is ruled out by its contribution to the electric dipole moment (EDM) of the neutron. However, contributions of the other Yukawas can cancel the contribution to EDMs without spoiling baryogenesis [2]. This motivates a detailed study of CP-violating contributions to all Yukawa couplings. 
In ref. [3], EDM constraints on the third generation (top, bottom, tau) Yukawa couplings were presented and compared to bounds from Higgs production and decay at the LHC (see ref. [4] for a more targeted collider study for the tau Yukawa). Presently, anomalous bottom Yukawas are more stringently constrained by $h \rightarrow b \bar{b}$ than by hadronic EDMs [3]. EDM and collider constraints on the electron Yukawa were subsequently studied in ref. [5]. A more generic approach was taken in a series of works [6-8], in which EDM constraints on Higgs-quark and Higgs-gluon couplings were studied in the SM effective field theory (EFT) approach. However, these analyses neglect logarithmically enhanced effects that were shown to be large in ref. [3], and two-loop Barr-Zee contributions to the light (up, down, and strange) Yukawas [9].

In the present work, we address CP violation in the bottom- and charm-quark Yukawas. The leading-logarithmic (LL) analysis for the case of the bottom quark was performed in ref. [3]. Although not discussed there, the residual perturbative uncertainty is significant, exceeding the one in the non-perturbative hadronic matrix elements. Recent progress in lattice determinations of these matrix elements [10-13] motivates a next-to-leadinglogarithmic (NLL) analysis in order to reduce the perturbative error of EDM predictions for the case of beyond-the-SM CP violation in the bottom and/or charm Yukawa. We calculate the complete anomalous dimension matrix for the mixing of CP-odd scalar and tensor operators up to next-to-leading (two-loop) order, and apply our results for a phenomenological study of CP violation in the bottom and charm Yukawa couplings.

This paper is organized as follows. In section 2 we define the effective theory needed for our calculation, and present the calculation of the renormalisation-group (RG) evolution of the Wilson coefficients in section 3. We illustrate the impact of our calculation on the constraints on the CP phases in section 4, and conclude in section 5. The appendices contain further details on our work. In section A we collect the requisite unphysical operators, in section $\mathrm{B}$ we present all relevant renormalisation constants, and in section $\mathrm{C}$ we present the full anomalous-dimension matrix. We discuss effect on our results of a change of the renormalisation scheme in section D. Section E contains the expansion of the resummed results.

\section{Effective theory below the weak scale}

Our starting point is a Lagrangian in which the Higgs particle couples to bottom or charm quarks differently than in the SM. Such a modification can originate from $\mathrm{TeV}$-scale new physics that can be parameterised by higher-dimensional operators, e.g., dimension-six operators of the form

$$
H^{\dagger} H \bar{Q}_{L, 3} H^{\dagger} d_{R, 3} .
$$

Here, $H$ denotes the Higgs doublet in the unbroken phase of electroweak gauge symmetry, while $Q_{L, 3}$ and $d_{R, 3}$ represent the left-handed quark doublet and the right-handed downquark field of the third generation, respectively.

The presence of such operators induces anomalous couplings of the Higgs particle to quarks in the electroweak broken phase. At leading order in the electroweak interactions the above dimension-six operators lead to a modification of the SM Yukawa interactions 
which, for the bottom quark, can be conveniently parameterised as

$$
\mathscr{L}_{h b b}=-\frac{y_{b}^{\mathrm{SM}}}{\sqrt{2}} \kappa_{b} \bar{b}\left(\cos \phi_{b}+i \gamma_{5} \sin \phi_{b}\right) b h .
$$

Here, $b$ denotes the bottom-quark field and $h$ the physical Higgs field. Moreover, $y_{b}^{\mathrm{SM}} \equiv$ $\frac{e}{\sqrt{2} s_{w}} \frac{m_{b}}{M_{W}}$ is the SM Yukawa, with $e$ the positron charge, $s_{w}$ the sine of the weak mixing angle, and $m_{b}$ and $M_{W}$ the bottom-quark and $W$-boson mass, respectively. The real parameter $\kappa_{b} \geq 0$ parameterises modifications to the absolute value of the Yukawa coupling, while the phase $\phi_{b} \in[0,2 \pi)$ parameterises $\mathrm{CP}$ violation and the sign of the Yukawa. The $\mathrm{SM}$ corresponds to $\kappa_{b}=1$ and $\phi_{b}=0$. Modifications of the charm-quark Yukawa can be parameterised in an analogous way.

The parametrisation of pseudoscalar Higgs couplings solely via the interaction in eq. (2.2) should be thought of as the dimension-four part of the so-called Higgs Effective Field Theory [14] or the electroweak chiral Lagrangian [15] in unitarity gauge for the electroweak sector. In this sense, the SM extended by a pseudoscalar Higgs coupling is not a renormalizable theory. However, as we consider in this work only loops induced by the strong coupling, we do not encounter additional divergences since the operators of the form (2.1) mix only into themselves under QCD [16]. This picture would change if one considered higher-order electroweak corrections.

The basic idea underlying this work is to calculate the effect of CP-odd phases in the Higgs Yukawa couplings of the bottom and charm quark on hadronic EDMs. EDMs receive contributions from partonic CP-violating electric and chromoelectric dipole operators, with coefficients $d_{q}$ and $\tilde{d}_{q}$. They are traditionally defined via the effective Lagrangian valid at hadronic energies $\mu \simeq 2 \mathrm{GeV}$ [17],

$$
\mathscr{L}_{\mathrm{eff}}=-d_{q} \frac{i}{2} \bar{q} \sigma^{\mu \nu} \gamma_{5} q F_{\mu \nu}-\tilde{d}_{q} \frac{i g_{s}}{2} \bar{q} \sigma^{\mu \nu} T^{a} \gamma_{5} q G_{\mu \nu}^{a}+\frac{1}{3} w f^{a b c} G_{\mu \sigma}^{a} G_{\nu}^{b, \sigma} \widetilde{G}^{c, \mu \nu},
$$

with $\sigma^{\mu \nu}=\frac{i}{2}\left[\gamma^{\mu}, \gamma^{\nu}\right]$, as well as $T^{a}$ the fundamental generators of $\mathrm{SU}\left(n_{c}\right)$ with $\operatorname{Tr}\left[T^{a}, T^{b}\right]=$ $\delta^{a b} / 2$ and $n_{c}=3$ the number of colors. This Lagrangian also includes the purely gluonic Weinberg operator [18]. Its contributions are subdominant because of its small nuclear matrix elements $[17,19]$, but are kept for completeness.

CP-violating Yukawa couplings contribute to $d_{q}$ and $\tilde{d}_{q}$ via Barr-Zee-type diagrams [20] with heavy-quark loops, see figure 1 . The Weinberg operator is induced via a finite threshold correction [21, 22]. In the case of a CP-violating bottom Yukawa, expanding the loop functions for small bottom mass and matching directly onto the Lagrangian of eq. (2.3), we find

$$
\begin{aligned}
d_{q} & \simeq-12 e Q_{q} Q_{b}^{2} \frac{\alpha}{(4 \pi)^{3}} \sqrt{2} G_{F} m_{q} \kappa_{b} \sin \phi_{b} x_{b}\left(\log ^{2} x_{b}+\frac{\pi^{2}}{3}\right), \\
\tilde{d}_{q} & \simeq 2 \frac{\alpha_{s}}{(4 \pi)^{3}} \sqrt{2} G_{F} m_{q} \kappa_{b} \sin \phi_{b} x_{b}\left(\log ^{2} x_{b}+\frac{\pi^{2}}{3}\right), \\
w & \simeq-g_{s} \frac{\alpha_{s}}{(4 \pi)^{3}} \sqrt{2} G_{F} x_{b} \kappa_{b}^{2} \cos \phi_{b} \sin \phi_{b}\left(\frac{3}{2}+\log x_{b}\right),
\end{aligned}
$$

up to higher orders in $x_{b} \equiv m_{b}^{2} / M_{h}^{2}$. 

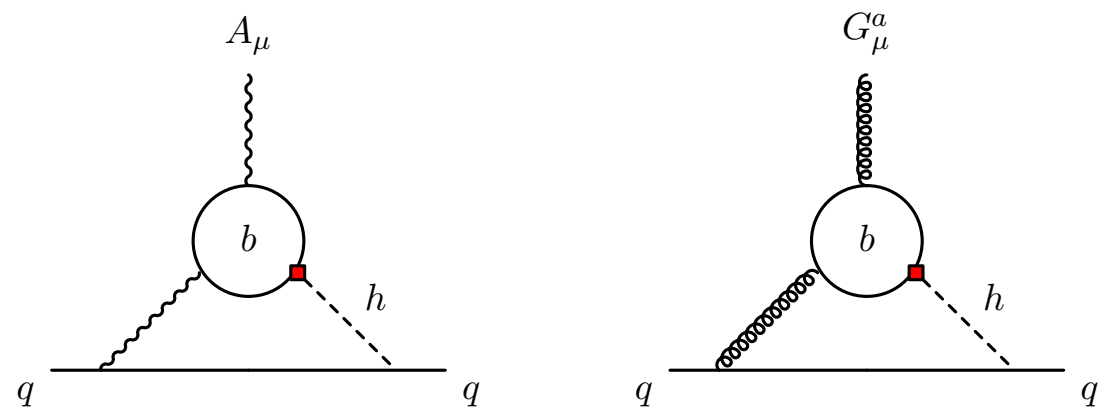

Figure 1. Photonic and gluonic "Barr-Zee" diagrams with modified bottom-Yukawa coupling that induce an EDM of the light quark $q$. See text for details.

However, as already noted in ref. [3], such a naive evaluation of the gluonic diagram leads to an uncertainty of a factor of order five. The uncertainty is related to the ambiguity in choosing the proper value of the strong coupling $\alpha_{s}(\mu)$; namely, at which dynamical scale should it be evaluated - the weak scale, the bottom-quark mass, or the hadronic scale? This scale dependence is related to logarithms of the large scale ratios and can be reduced by resummation of the large logarithms, which is easiest performed in an effective theory (EFT) framework. The LL series then reproduces the quadratic logarithm in eq. (2.4), while also resumming all higher-order terms. The uncertainties after the LL resummation are still large, at the order of two at the Wilson coefficient level. Hence, in this work we extend the LL analysis of ref. [3] to NLL and discuss the remaining theory uncertainty via the residual scale and scheme dependence in detail. In addition, we consider also modifications of the charm-quark Yukawa.

To construct the effective Lagrangian originating from anomalous flavor-conserving, CP-violating Higgs couplings to quarks we integrate out the heavy degrees of freedom of the SM (the top quark, the weak gauge bosons, and the Higgs) and match onto an effective five-flavor Lagrangian. EDMs are then induced by non-renormalizable operators that are $\mathrm{CP}$ odd. The corresponding Lagrangian reads

$$
\mathscr{L}_{\text {eff }}=-\sqrt{2} G_{F}\left(\sum_{q \neq q^{\prime}}\left[\sum_{i=1,2} C_{i}^{q q^{\prime}} O_{i}^{q q^{\prime}}+\frac{1}{2} \sum_{i=3,4} C_{i}^{q q^{\prime}} O_{i}^{q q^{\prime}}\right]+\sum_{q} \sum_{i=1, \ldots, 4} C_{i}^{q} O_{i}^{q}+C_{w} O_{w}\right),
$$

where the sums run over all quarks with masses below the weak scale $\left(q, q^{\prime}=u, d, s, c, b\right)$. The linearly independent operators are

$$
\begin{array}{ll}
O_{1}^{q q^{\prime}} & =(\bar{q} q)\left(\bar{q}^{\prime} i \gamma_{5} q^{\prime}\right), \\
O_{3}^{q q^{\prime}} & =\frac{1}{2} \epsilon^{\mu \nu \rho \sigma}\left(\bar{q} \sigma_{\mu \nu} q\right)\left(\bar{q}^{\prime} \sigma_{\rho \sigma} q^{\prime}\right), \\
O_{4}^{q q^{\prime}} & =\frac{1}{2} \epsilon^{\mu \nu \rho \sigma}\left(\bar{q} \sigma_{\mu \nu} T^{a} q\right)\left(\bar{q}^{\prime} \sigma_{\rho \sigma} T^{a} q^{\prime}\right),
\end{array}
$$




$$
\begin{array}{ll}
O_{1}^{q}=(\bar{q} q)\left(\bar{q} i \gamma_{5} q\right), & O_{2}^{q}=\frac{1}{2} \epsilon^{\mu \nu \rho \sigma}\left(\bar{q} \sigma_{\mu \nu} q\right)\left(\bar{q} \sigma_{\rho \sigma} q\right), \\
O_{3}^{q}=\frac{e Q_{q}}{2} \frac{m_{q}}{g_{s}^{2}} \bar{q} \sigma^{\mu \nu} q \tilde{F}_{\mu \nu}, & O_{4}^{q}=-\frac{1}{2} \frac{m_{q}}{g_{s}} \bar{q} \sigma^{\mu \nu} T^{a} q \tilde{G}_{\mu \nu}^{a}
\end{array}
$$

and

$$
O_{w}=-\frac{1}{3 g_{s}} f^{a b c} G_{\mu \sigma}^{a} G_{\nu}^{b, \sigma} \widetilde{G}^{c, \mu \nu}
$$

The basis of all CP-odd operators is closed under the renormalisation group flow of both QCD and QED as they both conserve CP.

In these equations, the $\gamma_{5}$ matrix is defined by

$$
\gamma_{5}=\frac{i}{4 !} \epsilon_{\mu \nu \rho \sigma} \gamma^{\mu} \gamma^{\nu} \gamma^{\rho} \gamma^{\sigma}
$$

where $\epsilon^{\mu \nu \rho \sigma}$ is the totally antisymmetric Levi-Civita tensor in four space-time dimensions with $\epsilon_{0123}=-\epsilon^{0123}=1$, and we use the notation $\widetilde{G}^{a, \mu \nu}=\frac{1}{2} \epsilon^{\mu \nu \rho \sigma} G_{\rho \sigma}^{a}$. We have included the factor $1 / 2$ in the contributions of the $O_{3(4)}^{q q^{\prime}}$ operators to the effective Lagrangian to account for the double counting implied by the sums in eq. (2.5). The non-standard sign convention for $O_{3}^{q}$ is related to our definition of the covariant derivative, eq. (A.3).

Note that whenever possible we defined the operators directly in terms of the LeviCivita tensors instead of $\gamma_{5}$ matrices. This reduces the number of $\gamma$ matrices entering the computation of the anomalous dimensions. In $d=4$ dimensions our definition is equivalent to the usual one because of the relation

$$
\sigma^{\mu \nu} \gamma_{5} \stackrel{[d=4]}{=}-\frac{i}{2} \epsilon^{\mu \nu \rho \sigma} \sigma_{\rho \sigma}
$$

valid in $d=4$. While such evanescent differences in the definition of the operators do not affect the leading-order anomalous dimensions, they do affect the next-to-leading-order results that we compute in this work. For further details regarding our treatment of $\gamma_{5}$ see section 3.2 .

\section{Renormalisation group evolution}

Our goal is the summation of all leading and next-to-leading logarithms via RG improved perturbation theory in the five-, four-, and three-flavor effective theory. The calculation proceeds in several steps. First, we integrate out the Higgs and weak gauge bosons together with the top quark at the electroweak scale, $\mu \sim M_{h}=125.10 \mathrm{GeV}$. After the RG flow, the heavy (bottom and charm) quarks are integrated out at their respective masses, $m_{b}\left(m_{b}\right)=$ $4.18 \mathrm{GeV}$ and $m_{c}\left(m_{c}\right)=1.27 \mathrm{GeV}$ (all numerical input values are taken from ref. [23]). We then match to an effective three-flavor theory where only the light-quark operators are present. Finally, we evaluate the Wilson coefficients in the three-flavor theory at $\mu_{\text {str }}=2 \mathrm{GeV}$ where the hadronic matrix elements of the electric dipole operators are given by the lattice calculations. The RG evolution between the different matching scales 

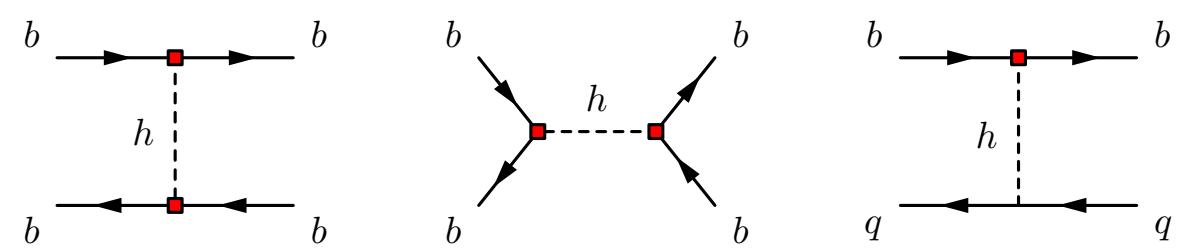

Figure 2. Sample tree-level Feynman diagrams contributing to the calculation of the initial conditions of the RG evolution at the electroweak scale for the case of modified bottom-quark Yukawas (indicated by the red square). Light quarks are denoted by the label $q=u, d, s$.

is computed using the appropriate anomalous dimensions, following the general formalism outlined in ref. [24].

The actual calculation was performed with self-written FORM [25] routines, implementing the two-loop recursion presented in refs. [26, 27]. The amplitudes were generated using QGRAF [28].

\subsection{Initial conditions at the weak scale}

We augment the SM with flavor-conserving anomalous Higgs Yukawas parameterised as in eq. (2.2) and at a scale $\mu_{\mathrm{ew}} \approx M_{h}$ integrate out the heavy degrees of freedom of the SM. Up to quadratic order in the strong coupling constant, we find the following non-zero initial conditions for the Wilson coefficients at scale $\mu_{\text {ew }}$ :

$$
\begin{aligned}
C_{1}^{q q^{\prime}}\left(\mu_{\mathrm{ew}}\right) & =-\kappa_{q} \kappa_{q^{\prime}} \cos \phi_{q} \sin \phi_{q^{\prime}} \frac{m_{q} m_{q^{\prime}}}{M_{h}^{2}}+\mathcal{O}\left(\alpha_{s}^{2}\right) \\
C_{4}^{q q^{\prime}}\left(\mu_{\mathrm{ew}}\right) & =\frac{\alpha_{s}}{4 \pi}\left(\frac{3}{2}+\log \frac{\mu_{\mathrm{ew}}^{2}}{M_{h}^{2}}\right) \frac{m_{q} m_{q^{\prime}}}{M_{h}^{2}} \kappa_{q} \kappa_{q^{\prime}}\left(\cos \phi_{q} \sin \phi_{q^{\prime}}+\sin \phi_{q} \cos \phi_{q^{\prime}}\right)+\mathcal{O}\left(\alpha_{s}^{2}\right) \\
C_{1}^{q}\left(\mu_{\mathrm{ew}}\right) & =-\left(1+\frac{\alpha_{s}}{4 \pi}\left(\frac{9}{2}+3 \log \frac{\mu_{\mathrm{ew}}^{2}}{M_{h}^{2}}\right)\right) \frac{m_{q}^{2}}{M_{h}^{2}} \kappa_{q}^{2} \cos \phi_{q} \sin \phi_{q}+\mathcal{O}\left(\alpha_{s}^{2}\right) \\
C_{2}^{q}\left(\mu_{\mathrm{ew}}\right) & =\frac{\alpha_{s}}{4 \pi}\left(\frac{1}{8}+\frac{1}{12} \log \frac{\mu_{\mathrm{ew}}^{2}}{M_{h}^{2}}\right) \frac{m_{q}^{2}}{M_{h}^{2}} \kappa_{q}^{2} \cos \phi_{q} \sin \phi_{q}+\mathcal{O}\left(\alpha_{s}^{2}\right) \\
C_{3}^{q}\left(\mu_{\mathrm{ew}}\right) & =-\frac{\alpha_{s}}{4 \pi}\left(3+2 \log \frac{\mu_{\mathrm{ew}}^{2}}{M_{h}^{2}}\right) \frac{m_{q}^{2}}{M_{h}^{2}} \kappa_{q}^{2} \cos \phi_{q} \sin \phi_{q}+\mathcal{O}\left(\alpha_{s}^{2}\right) \\
C_{4}^{q}\left(\mu_{\mathrm{ew}}\right) & =-\frac{\alpha_{s}}{4 \pi}\left(3+2 \log \frac{\mu_{\mathrm{ew}}^{2}}{M_{h}^{2}}\right) \frac{m_{q}^{2}}{M_{h}^{2}} \kappa_{q}^{2} \cos \phi_{q} \sin \phi_{q}+\mathcal{O}\left(\alpha_{s}^{2}\right)
\end{aligned}
$$

We see that if a single CP-violating Yukawa is switched on, e.g., the $q$-Yukawa with $q=b, c$, then at tree-level only the operators $O_{1}^{q}$ and $O_{1}^{q^{\prime} q}$ are induced by the anomalous Higgs coupling to $q$, i.e., to bottom or charm quarks, see figure 2. At one loop, also the operators $O_{2 \ldots 4}^{q}$ and $O_{4}^{q q^{\prime}}$ receive non-zero initial conditions (figure 3 ).

The quark masses in the expressions above are understood to be evaluated at the matching scale $\mu=\mu_{\text {ew }}$. Note that the one-loop expressions depend on the definition of evanescent operators; our choice is given in appendix A. Our predictions of physical ob- 

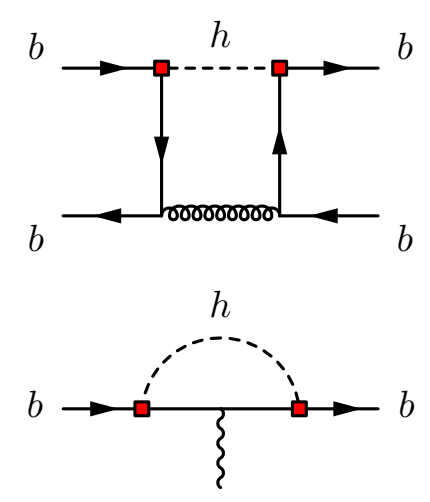

$A_{\mu}$
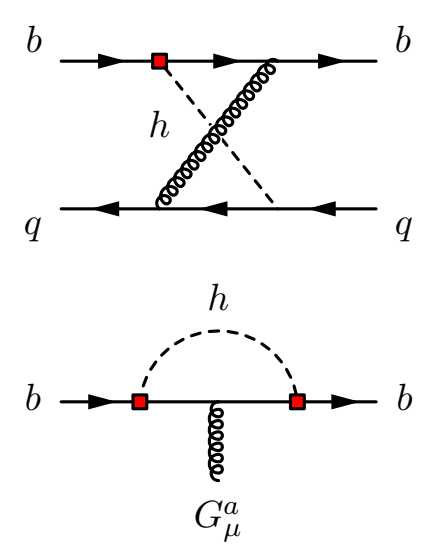

Figure 3. Sample one-loop Feynman diagrams contributing to the calculation of the initial conditions of the RG evolution at the electroweak scale for the case of modified bottom-quark Yukawas (indicated by the red square). Light quarks are denoted by the label $q=u, d, s$.

servables does not depend on the renormalisation scheme to $\mathcal{O}\left(\alpha_{s}\right)$; we discuss the residual scheme dependence in section 4 .

\subsection{Calculation of the anomalous dimensions}

The RG evolution below the weak scale in the presence of the heavy quarks can be calculated from the mixing of the operators in eq. (2.5). The one-loop results are fully known (see, e.g., ref. [29]). The two-loop mixing of the dipole operators has been presented in refs. [30, 31]. As a cross check we have reproduced all these results in the literature (see appendix D for details). The two-loop mixing of the four-fermion operators among themselves and into the dipole operators is presented here for the first time.

We calculate the anomalous dimensions by extracting the divergent parts of the insertions of all operators into appropriate Greens functions (see figure 4 for sample diagrams involving the bottom quark). We use dimensional regularisation, working in $d=4-2 \epsilon$ space-time dimensions, and employ the method of infrared rearrangement (IRA) described in ref. [32] to disentangle infrared from ultraviolet poles. For the whole computation we work in generic $R_{\xi_{g}}$ gauge for $\mathrm{SU}(3)_{c}$, which provides further checks for the correctness of the computation.

The appearance of $\gamma_{5}$ in closed fermion loops requires special care, since the use of a naively anticommuting $\gamma_{5}$, with $\left\{\gamma^{\mu}, \gamma_{5}\right\}=0$ for all $\mu$ (NDR scheme) is algebraically inconsistent if traces with $\gamma_{5}$ have to be evaluated [33]. Since such traces appear in our calculation we use Larin's prescription [34] throughout: the Levi-Civita tensor appearing either in the definition of $\gamma_{5}$ or in the effective operators is taken outside the momentum integrals and acts as a projector on the four-dimensional physical subspace after the momentum integration has been performed. The momentum integration, including the Dirac algebra, is then performed in $d$ space-time dimensions. The contraction of the Levi-Civita tensor with the results of the momentum integrals generates several evanescent operators that affect the two-loop anomalous dimensions in the usual way; they are listed in appendix A. No 
more than one Levi-Civita tensor appears in any diagram of the computation, so there is no need to contract multiple tensors.

The RG flow of the Wilson coefficients is governed by the RG equation

$$
\frac{d C^{t}}{d \log \mu}=C^{t} \gamma \quad \text { with } \quad \gamma=\frac{\alpha_{s}}{4 \pi} \gamma^{(0)}+\left(\frac{\alpha_{s}}{4 \pi}\right)^{2} \gamma^{(1)}+\ldots,
$$

where the superscript $t$ denotes transposition and $\gamma^{(0)}, \gamma^{(1)}$ are the one- and two-loop anomalous-dimension matrices, respectively. For our NLL analysis we require the two-loop mixing of the four-quark operators among themselves and into the dipole operators.

Below we collectively use the subscripts $q q^{\prime}$ and $q$ in the $\gamma$ 's to indicate subblocks of the full anomalous-dimension matrix. The ordering in each case is

$$
C_{q q^{\prime}}=\left\{C_{1}^{q q^{\prime}}, C_{2}^{q q^{\prime}}, C_{1}^{q^{\prime} q}, C_{2}^{q^{\prime} q}, C_{3}^{q q^{\prime}}, C_{4}^{q q^{\prime}}\right\}, \quad \text { and } \quad C_{q}=\left\{C_{1}^{q}, C_{2}^{q}, C_{3}^{q}, C_{4}^{q}\right\} .
$$

With the definition of evanescent operators given in appendix A, we find the mixing among four-fermion operators with two different flavors to be

$$
\gamma_{q q^{\prime} \rightarrow q q^{\prime}}^{(1)}=\left(\begin{array}{cccc}
-\frac{16 N_{f}}{3}-36 & -\frac{5}{2} & -\frac{28}{3} \\
-\frac{5}{9} & \frac{1045}{12}-\frac{16 N_{f}}{3} & -\frac{35}{9} \\
-\frac{28}{3} & -\frac{35}{2} & -\frac{16 N_{f}}{3}-36 \\
-\frac{35}{9} & \frac{1}{4} & -\frac{5}{9} \\
0 & \frac{16 N_{f}}{3}-24 & 0 \\
\frac{32 N_{f}}{27}-\frac{496}{3} & \frac{20 N_{f}}{9}-190 & \frac{32 N_{f}}{27}-\frac{496}{3} \\
& -\frac{35}{2} & 0 & \frac{2 N_{f}}{9}-37 \\
\frac{1}{4} & \frac{4 N_{f}}{81}+\frac{10}{9} & \frac{5 N_{f}}{54}-\frac{95}{12} \\
\frac{1045}{12}-\frac{16 N_{f}}{3} & \frac{4 N_{f}}{81}+\frac{10}{9} & \frac{5 N_{f}}{54}-\frac{95}{12} \\
\frac{16 N_{f}}{3}-24 & \frac{1288}{9}-\frac{208 N_{f}}{27} & -\frac{100}{3} \\
\frac{20 N_{f}}{9}-190 & -\frac{200}{27} & -\frac{100 N_{f}}{27}-\frac{340}{9}
\end{array}\right) .
$$

We find for their mixing into four-quark operators with one quark flavor and into dipoles

$$
\gamma_{q q^{\prime} \rightarrow q}^{(1)}=\left(\begin{array}{cccc}
0 & 0 & 0 & -12 \frac{m_{q^{\prime}}}{m_{q}} \\
0 & 0 & -16 \frac{Q_{q^{\prime}}}{Q_{q}} \frac{m_{q^{\prime}}}{m_{q}} & -5 \frac{m_{q^{\prime}}}{m_{q}} \\
0 & 0 & 0 & -4 \frac{m_{q^{\prime}}}{m_{q}} \\
0 & 0 & -\frac{16}{3} \frac{Q_{q^{\prime}}}{Q_{q}} \frac{m_{q^{\prime}}}{m_{q}} & -\frac{5}{3} \frac{m_{q^{\prime}}}{m_{q}} \\
0 & 0 & -448 \frac{Q_{q^{\prime}}}{Q_{q}} \frac{m_{q^{\prime}}}{m_{q}} & 0 \\
0 & 0 & \frac{128}{3} \frac{m_{q^{\prime}}}{m_{q}} & -92 \frac{m_{q^{\prime}}}{m_{q}}
\end{array}\right) .
$$



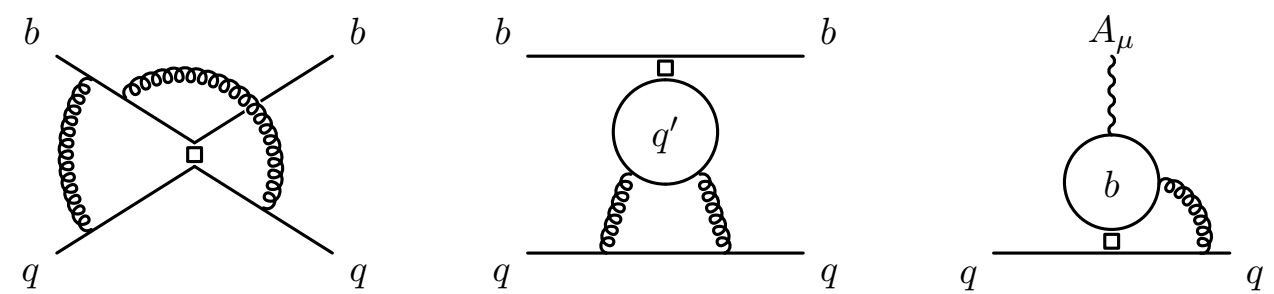

Figure 4. Sample two-loop Feynman diagrams whose divergent parts contribute to the calculation of the two-loop anomalous dimensions involving bottom quarks. The empty squares symbolize the insertion of an effective operator. The diagrams of the kind shown in the middle panel, involving three different quark flavors, vanish due to the odd number of Dirac matrices in the trace. Diagrams of the kind shown in the right panel contain traces of Dirac matrices with $\gamma_{5}$, which are treated in the Larin scheme.

For the anomalous dimensions in the $q \rightarrow q$ sector we find

$$
\gamma_{q \rightarrow q}^{(1)}=\left(\begin{array}{cccc}
65-6 N_{f} & \frac{N_{f}}{54}-\frac{19}{12} & \frac{848}{27} & \frac{1235}{27} \\
60-\frac{40 N_{f}}{9} & \frac{403}{3}-\frac{226 N_{f}}{27} & -\frac{6976}{9} & -\frac{820}{9} \\
0 & 0 & \frac{460 N_{f}}{27}+\frac{100}{9} & 0 \\
0 & 0 & 112-\frac{128 N_{f}}{27} & \frac{449 N_{f}}{27}+\frac{577}{9}
\end{array}\right)
$$

The two-loop mixing among the dipole operators $O_{3}^{q}$ and $O_{4}^{q}$ has been calculated before; the relation between our results and the literature is discussed in appendix D. All remaining results are new.

The two-loop ADM that involve the Weinberg operator and do not necessarily vanish are $\gamma_{W \rightarrow q}^{(1)}, \gamma_{W \rightarrow q q^{\prime}}^{(1)}$, and $\gamma_{W \rightarrow W}^{(1)}$. They are not needed in our analysis, since the Weinberg operator contributes only via a finite threshold correction, see section 3.3 (the two-loop and three-loop self mixings of the Weinberg operator for the case of $N_{f}=0$ have recently been published for pure Yang-Mills theory in ref. [35]). All remaining subblocks are zero, i.e.,

$$
\gamma_{q \rightarrow q^{\prime}}^{(1)}=\gamma_{q \rightarrow q q^{\prime}}^{(1)}=\gamma_{q \rightarrow q^{\prime} q^{\prime \prime}}^{(1)}=\gamma_{q q^{\prime} \rightarrow q^{\prime \prime}}^{(1)}=\gamma_{q q^{\prime} \rightarrow q^{\prime} q^{\prime \prime}}^{(1)}=\gamma_{q q^{\prime} \rightarrow q^{\prime \prime} q^{\prime \prime \prime}}^{(1)}=\gamma_{q \rightarrow W}^{(1)}=\gamma_{q q^{\prime} \rightarrow W}^{(1)}=\mathbf{0}
$$

The appearance of quark-mass ratios in these matrices is related to the explicit factors of quark masses in eq. (2.10). These mass ratios are scale- and scheme independent to the order we are working. They could, in principle, be avoided altogether by defining several dipole operators with the same field content, but different quark mass factors.

For completeness, we collect the one-loop anomalous dimensions in appendix C.

\subsection{Matching at the heavy-quark thresholds}

If $m_{q} \ll m_{q^{\prime}}$, the dipole operators with external $q$-quark lines receive matching corrections at the $q^{\prime}$-quark threshold. (In practice, $q^{\prime}=b$ or $q^{\prime}=c$.) We write the effective Lagrangian 
of the theory in which all heavy $q^{\prime}$ quarks have been integrated out as

$$
\mathscr{L}_{\text {eff }}=-\sqrt{2} G_{F}\left(\sum_{q} \sum_{i=3,4} C_{i}^{q} O_{i}^{q}+C_{w} O_{w}+\ldots\right) .
$$

Here, $q=u, d, s$ denotes one of the light quarks. CP-violating four-fermion operators involving only light quarks are present in principle and denoted by the ellipsis; however, their Wilson coefficients are suppressed by a power of $m_{q} / m_{q^{\prime}}$.

Let us call the theory with and without the $q^{\prime}$ quark the $f$-flavor and $(f-1)$-flavor theories, respectively. At the threshold scale $\mu_{f}$ the amplitudes in both theories must match each other; we write the equality between two general amplitudes in these theories as

$$
\sum_{i} C_{i, f}\left(\mu_{f}\right)\left\langle O_{i}\right\rangle_{f}\left(\mu_{f}\right)=\sum_{i} C_{i, f-1}\left(\mu_{f}\right)\left\langle O_{i}\right\rangle_{f-1}\left(\mu_{f}\right) .
$$

Here, angle brackets denote appropriate matrix elements. We expand the Wilson coefficients and matrix elements in the strong coupling of the $f$-flavor theory:

$$
\begin{aligned}
C_{i, f}\left(\mu_{f}\right) & =C_{i, f}^{(0)}+\frac{\alpha_{s}\left(\mu_{f}\right)}{4 \pi} C_{i, f}^{(1)}+\ldots, \\
\left\langle O_{i}\right\rangle_{f}\left(\mu_{f}\right) & =\sum_{j}\left(\delta_{i j}+\frac{\alpha_{s}\left(\mu_{f}\right)}{4 \pi} r_{i j, f}^{(1)}+\ldots\right)\left\langle O_{j}\right\rangle_{f}^{(0)}\left(\mu_{f}\right),
\end{aligned}
$$

where the ellipses denote higher orders in the strong coupling constant. We expressed the higher-order matrix elements in terms of tree-level matrix elements, denoted by the superscript "(0)", via the coefficients $r_{i j}$.

There are various subtleties to keep in mind when calculating the threshold corrections. The strong coupling constant itself receives a non-vanishing threshold correction at one loop,

$$
\alpha_{s}^{(f)}\left(\mu_{f}\right)=\alpha_{s}^{(f-1)}\left(\mu_{f}\right)\left(1+\frac{\alpha_{s}^{(f-1)}\left(\mu_{f}\right)}{4 \pi} \frac{2}{3} \log \frac{\mu_{f}^{2}}{m_{q^{\prime}}^{2}}+\ldots\right) .
$$

Similarly, the gluon field renormalisation receives a non-zero threshold correction. Finally, the anomalous dimensions of the dipole operators depend explicitly on the number of active quark flavors. The quark masses, on the other hand, are affected only at the two-loop level (see, e.g., ref. [36]).

By explicit calculation of various one-loop diagrams (see figure 5), we find, evaluating eq. (3.14), the following non-zero threshold corrections

$$
\begin{aligned}
C_{3, f-1}^{q,(1)} & =C_{3, f}^{q,(1)}+\left(24 C_{3, f}^{q q^{\prime},(0)}\left(\mu_{f}\right) \frac{m_{q^{\prime}}}{m_{q}} \frac{Q_{q^{\prime}}}{Q_{q}}-\frac{2}{3} C_{3, f}^{q,(0)}\left(\mu_{f}\right)\right) \log \frac{\mu_{f}^{2}}{m_{q^{\prime}}^{2}}, \\
C_{4, f-1}^{q,(1)} & =C_{4, f}^{q,(1)}+\left(4 C_{4, f}^{q q^{\prime},(0)}\left(\mu_{f}\right) \frac{m_{q^{\prime}}}{m_{q}}-\frac{2}{3} C_{4, f}^{q,(0)}\left(\mu_{f}\right)\right) \log \frac{\mu_{f}^{2}}{m_{q^{\prime}}^{2}}, \\
C_{w, f-1}^{(1)} & =C_{w, f}^{(1)}-\frac{4}{3} C_{w, f}^{(0)}\left(\mu_{f}\right) \log \frac{\mu_{f}^{2}}{m_{q^{\prime}}^{2}}-\frac{1}{2} C_{4, f}^{q^{\prime},(0)}\left(\mu_{f}\right),
\end{aligned}
$$

as a power series in the strong coupling in the $(f-1)$-flavor theory. 

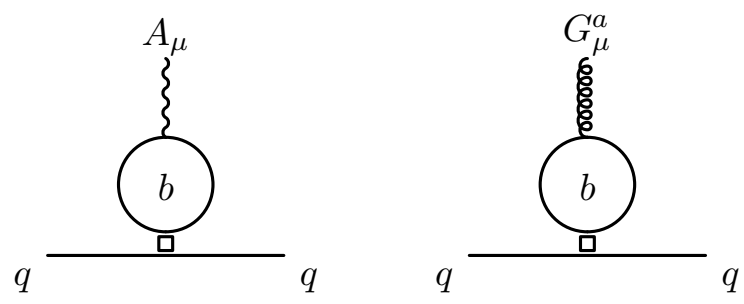

Figure 5. Sample Feynman diagrams whose finite parts contribute to the calculation of the matching corrections at the respective heavy-quark thresholds (shown here for the case of the bottom quark).

\section{Numerics}

In the last three sections we presented all the ingredients needed to consistently perform the resummation of large logarithms appearing in hadronic Barr-Zee-type diagrams from flavor-conserving CP-violating Higgs Yukawas at NLL: the next-to-leading-order (NLO) (one-loop) matching at the electroweak scale, the NLO (two-loop) anomalous-dimension matrix, and the (one-loop) threshold corrections for the Wilson coefficients at the heavyquark thresholds. In this section, we implement the NLL evolution numerically and discuss its impact on a set of hadronic EDMs. We first present values for the partonic Wilson coefficients in dependence of the $\mathrm{CP}$-violating phase and discuss the theoretical uncertainty in detail. Then we give bounds on the phases using experimental input.

\subsection{Wilson coefficients}

To compute the effect of modified Yukawa couplings on hadronic EDMs, we need the values of the induced Wilson coefficients of the electric dipole, chromoelectric dipole, and Weinberg operators at the scale $\mu_{\text {str }}=2 \mathrm{GeV}$ where the matrix elements of these operators are evaluated. We consider two cases: first, we only turn on a CP-violating bottom Yukawa and second, only a CP-violating charm Yukawa.

The dependence on the matching scales $\mu_{\mathrm{ew}}$ and $\mu_{b(c)}$ of the dipole Wilson coefficients evaluated at scale $\mu_{\mathrm{str}}=2 \mathrm{GeV}$ cancels at NLO in the expansion in $\alpha_{s} \cdot{ }^{1}$ However, the RG evolution induces a residual dependence on these scales. This dependence is formally of higher order in $\alpha_{s}$ and can be used to assess the remaining theoretical uncertainty of the prediction. In figure 6 we show the value of the dipole Wilson coefficients, evaluated at $2 \mathrm{GeV}$, as a function of the electroweak matching scale, $\mu_{\mathrm{ew}}$, varied within $M_{h} / 2 \leq \mu_{\text {ew }} \leq 2 M_{h}$. The Wilson coefficients contain terms proportional to either $\kappa_{b(c)} \sin \phi_{b(c)} / M_{h}^{2}$ or $\kappa_{b(c)}^{2} \sin \phi_{b(c)} \cos \phi_{b(c)} / M_{h}^{2}$. For the dipole operators, the latter terms are subleading. For purposes of illustration we thus choose to plot the case $\phi_{b(c)}=\pi / 2$, setting $\kappa_{b(c)}=1$ and factoring out the global Higgs-mass dependence. Focusing first on the Wilson coefficients $C_{3}^{q}$ of the electric dipole operators (red lines), we see that the scale dependence is both weak and barely reduced by going from LL to NLL. For the coefficients

\footnotetext{
${ }^{1}$ In our case, the Weinberg operator does not contribute at LL. Hence, there is no corresponding cancellation to the order we calculated.
} 


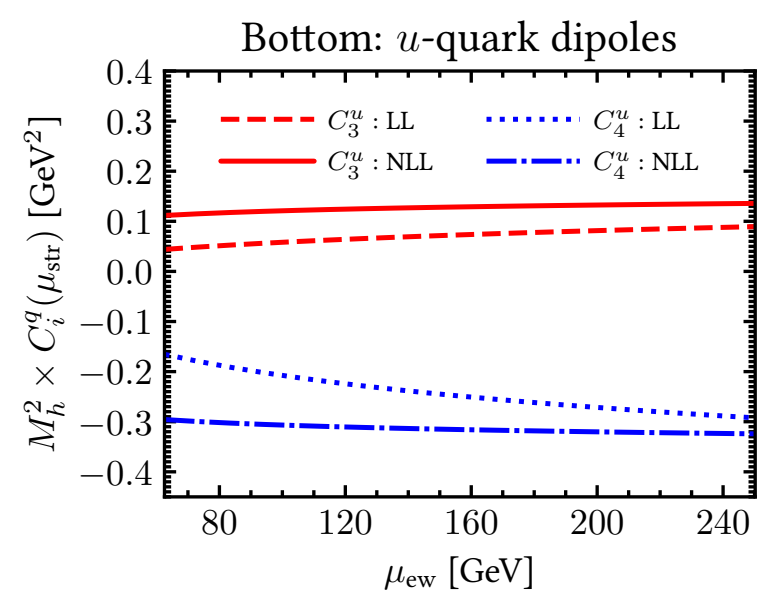

Bottom: $d / s$-quark dipoles
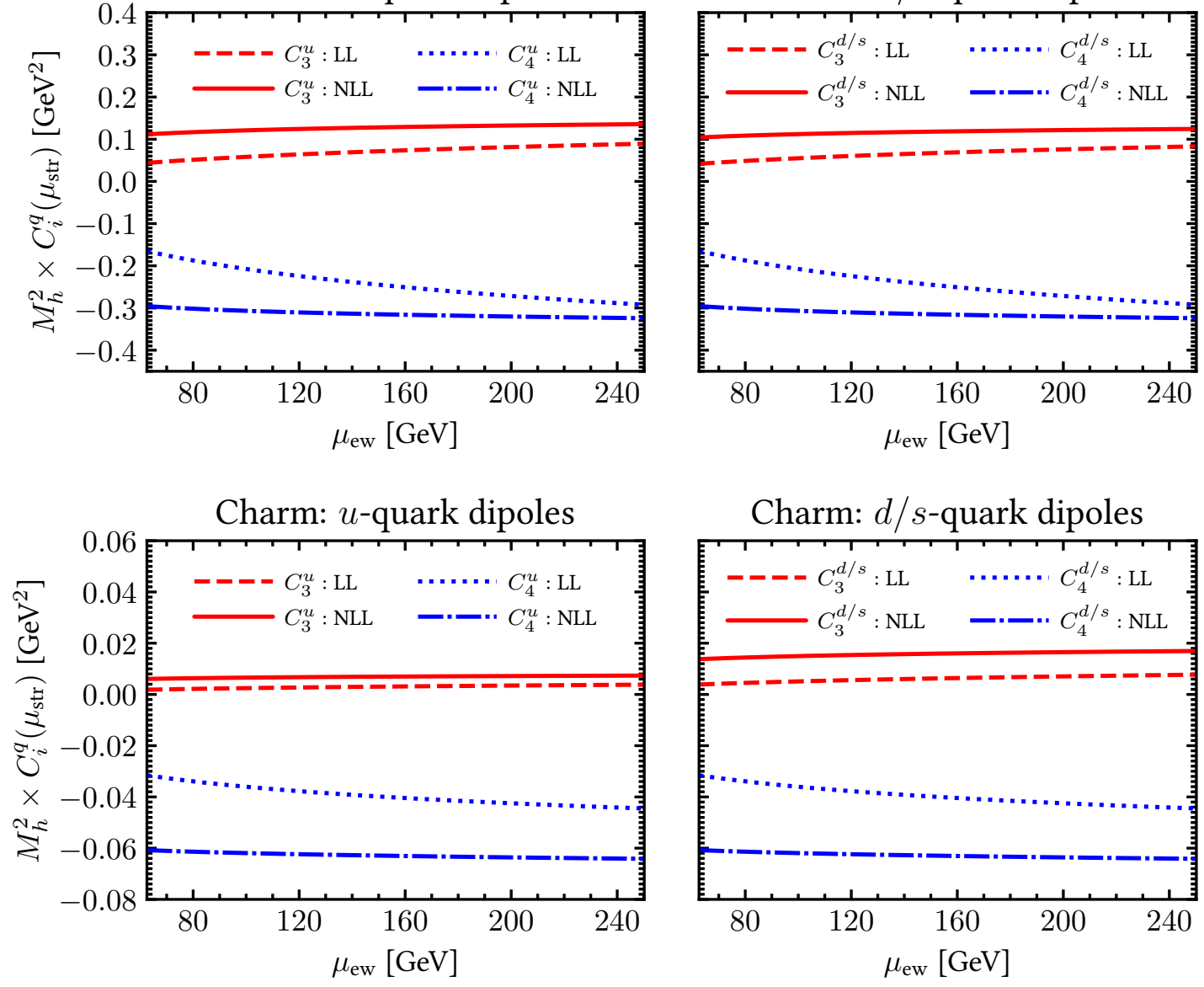

Figure 6. Residual dependence of the dipole Wilson coefficients on the electroweak matching scale. The upper two panels show the effect of a modified bottom Yukawa, the lower two panels the effect of a modified charm Yukawa. The left plots correspond to operators with external up quarks, while the right plot show operators with down-/strange-quark external legs. In all cases the Wilson coefficients are evaluated in the three-flavor theory at the hadronic scale $\mu_{\text {str }}=2 \mathrm{GeV}$, for $\phi_{b(c)}=\pi / 2$ and $\kappa_{b(c)}=1$.

$C_{4}^{q}$ of the chromoelectric dipole operator (blue lines) the scale dependence $\delta C_{4}^{q} / C_{4}^{q}$ is reduced by factor of approximately five. In all cases, however, the shift from the LL to the NLL results is substantial and larger than indicated by the residual scale dependence of both the LL and NLL result.

It is interesting to note that the RG evolution, in the case of a modified charm Yukawa, leads to a small Wilson coefficient of the electric dipole operator, such that the contribution to the hadronic EDMs at low scales is completely dominated by the chromoelectric dipole operator.

Next, we consider the residual dependence on the matching scale at which the heavy quark is integrated out. Similarly to figure 6 , in figure 7 we show the Wilson coefficients at $2 \mathrm{GeV}$, this time varying the heavy-quark matching scales within $2.5 \mathrm{GeV} \leq \mu_{b} \leq 10 \mathrm{GeV}$ 

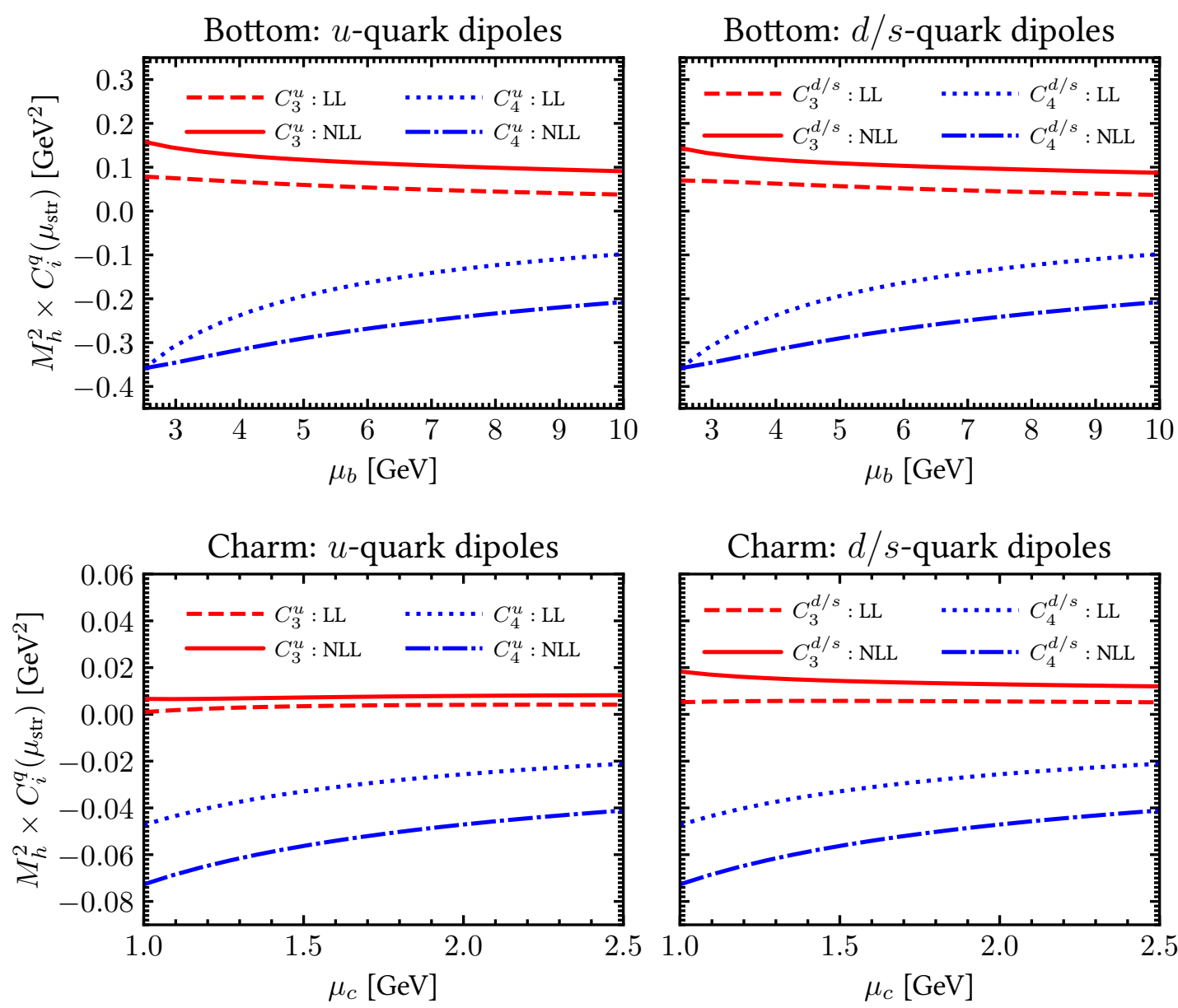

Figure 7. The same as figure 6, but the dependence on the matching scale at the bottom- and charm-quark thresholds is shown.

and $1 \mathrm{GeV} \leq \mu_{c} \leq 2.5 \mathrm{GeV}$. While the scale dependence of the electric dipole coefficients $C_{3}^{q}$ is still mild (red lines), the chromoelectric coefficients $C_{4}^{q}$ (blue lines) show a large residual scale dependence that is barely reduced in going from LL to NLL. Again, all Wilson coefficients show a large shift when including the NLL corrections.

While the large scale dependence at NLL can be partly understood by the appearance of many new, non-zero electroweak initial conditions at NLO (cf. eqs. (3.1)-(3.6)), the large shift is mainly due to the large numerical values of the entries in the NLO anomalousdimension matrix (see, e.g., eq. (3.10)). This suspicion is borne out by expanding the result of the RG evolution about the bottom-quark matching scale, illustrating the size of the higher-order corrections; see appendix E for the explicit results of this expansion.

One may then wonder whether these large entries in the anomalous-dimension matrix are an artefact of a badly chosen renormalisation scheme. In fact, as discussed for instance in refs. [37, 38], these entries depend on the definition of evanescent operators. Needless to say, this scheme dependence cancels up to the order to which the calculation is performed. 
Nevertheless, the residual scheme dependence can, in principle, be large. We have tested this by converting our anomalous dimension to different schemes chosen such that many of the large entries vanish. While the residual scale dependence is indeed somewhat smaller in these schemes, the central values of the Wilson coefficients strongly depend on the choice of scheme, taking values in approximately the same range as indicated by the scale dependence of the results in our original calculation.

All these observations hint at a slow convergence of the perturbation series. We therefore adopt the following prescription for the numerical values of the Wilson coefficients, including our best estimate of the associated remaining theory uncertainty: we obtain the "central values" of the Wilson coefficients as the value for $\mu_{\mathrm{ew}}=M_{h}$ and $\mu_{b(c)}=m_{b(c)}\left(m_{b(c)}\right)$ and assign as the theory uncertainty either half the range of the NLL scale variations, or half the shift between LL and NLL, whichever is larger.

We then find the three-flavour Wilson coefficients, evaluated at $2 \mathrm{GeV}$, of electric dipole, chromoelectric dipole, and Weinberg operators to be:

$$
\begin{aligned}
& C_{3, f=3}^{u}(2 \mathrm{GeV})=\left[\left(a_{3 u} \pm \Delta a_{3 u}\right) \kappa_{q} \sin \phi_{q}+\left(b_{3} \pm \Delta b_{3}\right) \kappa_{q}^{2} \sin \phi_{q} \cos \phi_{q}\right] \frac{\mathrm{GeV}^{2}}{M_{h}^{2}} \\
& C_{3, f=3}^{d, s}(2 \mathrm{GeV})=\left[\left(a_{3 d} \pm \Delta a_{3 d}\right) \kappa_{q} \sin \phi_{q}+\left(b_{3} \pm \Delta b_{3}\right) \kappa_{q}^{2} \sin \phi_{q} \cos \phi_{q}\right] \frac{\mathrm{GeV}^{2}}{M_{h}^{2}} \\
& C_{4, f=3}^{u, d, s}(2 \mathrm{GeV})=\left[\left(a_{4} \pm \Delta a_{4}\right) \kappa_{q} \sin \phi_{q}+\left(b_{4} \pm \Delta b_{4}\right) \kappa_{q}^{2} \sin \phi_{q} \cos \phi_{q}\right] \frac{\mathrm{GeV}^{2}}{M_{h}^{2}} \\
& C_{w, f=3}(2 \mathrm{GeV})=\left[\left(a_{w} \pm \Delta a_{w}\right) \kappa_{q} \sin \phi_{q}+\left(b_{w} \pm \Delta b_{w}\right) \kappa_{q}^{2} \sin \phi_{q} \cos \phi_{q}\right] \frac{\mathrm{GeV}^{2}}{M_{h}^{2}}
\end{aligned}
$$

Here, the subscripts $q=b, c$ refer to the cases of the bottom and charm quark, respectively. The values for the $a$ and $b$ coefficients and their respective uncertainties are given, for the two cases, in the second and third columns of table 1 . We see that in both cases the residual uncertainty on the dipole Wilson coefficients is of the order of $30 \%$, while the contributions induced by the Weinberg operator have larger uncertainties. They could potentially be reduced by a next-to-next-to-leading-logarithmic calculation.

\subsection{Bounds on CP phases from hadronic EDMs}

In this section we derive constraints on new CP-violating phases in the bottom or charm Yukawa under the simplifying assumption that only one such phase is present. A global fit for the general case including constraints from the LHC will be presented in a future publication [39].

We start with a generic parameterisation of a nuclear dipole moment, $X$, as

$$
d_{X}=d_{X}\left(f_{X, i}, a_{i}, b_{i}\right)
$$

Here, the $f_{X, i}$ with $i=1, \ldots, n$ are the hadronic input parameters entering the prediction of the given $d_{X}$. We denote their uncertainties by $\Delta f_{X, i}$. The $a_{i}$, with $i=3 u, 3 d, 4, w$, and $b_{i}$, with $i=3,4, w$, are defined in section 4.1 and given in table 1 . They parameterise 


\begin{tabular}{|rrr|}
\hline & \multicolumn{1}{c}{ Bottom Case } & \multicolumn{1}{c|}{ Charm Case } \\
\hline$a_{3 u} \pm \Delta a_{3 u}$ & $0.125 \pm 0.034$ & $(6.78 \pm 2.00) \times 10^{-3}$ \\
$a_{3 d} \pm \Delta a_{3 d}$ & $0.115 \pm 0.028$ & $(1.55 \pm 0.49) \times 10^{-2}$ \\
$a_{4} \pm \Delta a_{4}$ & $-0.311 \pm 0.076$ & $(-6.25 \pm 1.58) \times 10^{-2}$ \\
$a_{w} \pm \Delta a_{w}$ & $(4.10 \pm 2.50) \times 10^{-3}$ & $(4.10 \pm 2.05) \times 10^{-3}$ \\
$b_{3} \pm \Delta b_{3}$ & $(-1.66 \pm 1.35) \times 10^{-4}$ & $(-2.00 \pm 3.68) \times 10^{-5}$ \\
$b_{4} \pm \Delta b_{4}$ & $(1.84 \pm 0.36) \times 10^{-3}$ & $(-2.71 \pm 3.36) \times 10^{-4}$ \\
$b_{w} \pm \Delta b_{w}$ & $(-1.63 \pm 1.00) \times 10^{-2}$ & $(-3.41 \pm 1.69) \times 10^{-3}$ \\
\hline
\end{tabular}

Table 1. Numerical values for the coefficients $a$ and $b$ and their respective uncertainties as defined in eq. (4.1).

the Wilson coefficients that contribute to $d_{X}$ if either the bottom or the charm quark Yukawa is modified. By standard quadratic error propagation we compute the total theory uncertainty

$$
\Delta d_{X}^{\text {th }}=(\underbrace{\sum_{i=1}^{n}\left(\frac{\partial d_{X}}{\partial f_{X, i}} \Delta f_{X, i}\right)^{2}}_{\equiv\left(\Delta d_{X}^{\text {hadronic }}\right)^{2}}+\underbrace{\sum_{j=1}\left(\frac{\partial d_{X}}{\partial a_{j}} \Delta a_{j}\right)^{2}+\sum_{j=1}\left(\frac{\partial d_{X}}{\partial b_{j}} \Delta b_{j}\right)^{2}}_{\equiv\left(\Delta d_{X}^{\text {short-distance })^{2}}\right.})^{\frac{1}{2}} .
$$

To derive the allowed confidence level (CL) intervals from the measurements of dipole moments and to combine them we construct a $\chi^{2}$ function of two parameters, $\kappa_{q}, \phi_{q}$ or equivalently of $\kappa_{q} \sin \phi_{q}, \kappa_{q} \cos \phi_{q}$ :

$$
\chi^{2}\left(\kappa_{q}, \phi_{q}\right)=\sum_{X} \frac{\left(d_{X}^{\text {obs }}-d_{X}\right)^{2}}{\left(\Delta d_{X}^{\text {exp }}\right)^{2}+\left(\Delta d_{X}^{\text {hadronic }}\right)^{2}+\left(\Delta d_{X}^{\text {short-distance }}\right)^{2}},
$$

where we have neglected the tiny SM contribution to any EDM. The allowed $68.27 \%$ CL region for the two-parameters are then given by the region $\chi^{2}\left(\kappa_{q}, \phi_{q}\right)-\chi_{\min }^{2} \leq 2.30$.

The relation between the coefficients $d_{q}, \tilde{d}_{q}$, and $w$ in eq. (2.3) and the Wilson coefficients in the three-flavour EFT is

$$
\begin{aligned}
d_{q}(\mu) & =\sqrt{2} G_{F} \frac{Q_{q} e}{4 \pi \alpha_{s}} m_{q} C_{3}^{q}(\mu)-12 e Q_{q} Q_{b}^{2} \frac{\alpha}{(4 \pi)^{3}} \sqrt{2} G_{F} m_{q} \kappa_{b} \sin \phi_{b} x_{b}\left(\log ^{2} x_{b}+\frac{\pi^{2}}{3}\right), \\
\tilde{d}_{q}(\mu) & =-\sqrt{2} G_{F} \frac{1}{4 \pi \alpha_{s}} m_{q} C_{4}^{q}(\mu), \\
w(\mu) & =\sqrt{2} G_{F} \frac{1}{4 \pi \alpha_{s}} C_{w}(\mu) .
\end{aligned}
$$

In the expression for $d_{q}$ above we have included the electroweak contribution of eq. (2.4). From this we obtain the neutron EDM as

$$
\frac{d_{n}}{e}=(1.1 \pm 0.55)\left(\tilde{d}_{d}+0.5 \tilde{d}_{u}\right)+\left(g_{T}^{u} \frac{d_{u}}{e}+g_{T}^{d} \frac{d_{d}}{e}+g_{T}^{s} \frac{d_{s}}{e}\right) \pm(22 \pm 11) w \mathrm{MeV}
$$


where the matrix elements of the electric dipole operator are parameterised by $g_{T}^{u}=$ $-0.24(3), g_{T}^{d}=0.85(8), g_{T}^{s}=-0.012(18)$. These values are calculated using lattice QCD with $2+1$ active flavors and are converted to the $\overline{\mathrm{MS}}$ scheme at $2 \mathrm{GeV}[40,41]$ (see ref. [42] for a $N_{f}=2+1+1$ result). By using the lattice values of the three-flavour theory we include the effect of threshold corrections associated to the charm quark. To capture this effect in the four-flavour theory the matrix elements of four-quark operators with charm quarks would also need to be evaluated via lattice methods. The hadronic matrix elements of the chromoelectric dipole and the Weinberg operators are estimated using QCD sum rules and chiral techniques $[17,19]$. Notice that the sign of the hadronic matrix element of the Weinberg is not known, and thus the allowed CL intervals will depend on it. For prospects on obtaining the latter via lattice calculations see refs. [12, 13].

The experimental 90\% CL exclusion bound obtained in ref. [43] is $\left|d_{n}\right|<2.9 \times$ $10^{-26} e \mathrm{~cm}$. Using the central values of the Wilson coefficients in table 1 and the twoparameter $\chi^{2}$ we compute the allowed $68.27 \%$ CL intervals for the bottom- and charmquark cases. The label " $\operatorname{sign} w$ " indicates whether the sign of the Weinberg-operator contribution in eq. (4.6) is taken to be positive or negative. We show the CL intervals for the cases in which: i) no theory uncertainty is included (no theory error label), ii) only the short-distance theory uncertainty is included (with short-distance theory error label) iii) the short-distance theory uncertainty is added in quadrature with the present theory uncertainties of the hadronic input (with theory error label). For brevity we introduce the short-hand notation $\sin \phi_{b(c)} \equiv s_{b(c)}$ and $\cos \phi_{b(c)} \equiv c_{b(c)}$. For the bottom case we find the allowed $68.27 \%$ CL regions to be:

$$
\begin{aligned}
& \kappa_{b}\left|s_{b}\right| \sqrt{1+0.40 \kappa_{b} c_{b}+0.040 \kappa_{b}^{2} c_{b}^{2}} \leq 3.5 \quad\left[\operatorname{sign}_{w}=-, \text { no theory error }\right], \\
& \kappa_{b}\left|s_{b}\right| \sqrt{1+0.49 \kappa_{b} c_{b}+0.0037 \kappa_{b}^{2} c_{b}^{2}} \leq 3.8 \quad\left[\operatorname{sign}_{w}=-, \text { with short-distance theory error }\right], \\
& \kappa_{b}\left|s_{b}\right| \sqrt{1+5.4 \kappa_{b} c_{b}-0.27 \kappa_{b}^{2} c_{b}^{2}} \leq 12 \quad\left[\operatorname{sign}_{w}=-, \text { with theory error }\right], \\
& \kappa_{b}\left|s_{b}\right| \sqrt{1-0.39 \kappa_{b} c_{b}+0.037 \kappa_{b}^{2} c_{b}^{2}} \leq 3.1 \quad\left[\operatorname{sign}_{w}=+, \text { no theory error }\right], \\
& \kappa_{b}\left|s_{b}\right| \sqrt{1-0.45 \kappa_{b} c_{b}+0.0082 \kappa_{b}^{2} c_{b}^{2}} \leq 3.4 {\left[\operatorname{sign}_{w}=+, \text { with short-distance theory error }\right], } \\
& \kappa_{b}\left|s_{b}\right| \sqrt{1-1.5 \kappa_{b} c_{b}-0.054 \kappa_{b}^{2} c_{b}^{2}} \leq 6.4 \quad\left[\operatorname{sign}_{w}=+, \text { with theory error }\right] .
\end{aligned}
$$

For the charm case we find:

$$
\begin{aligned}
& \kappa_{c}\left|s_{c}\right| \sqrt{1+0.53 \kappa_{c} c_{c}+0.069 \kappa_{c}^{2} c_{c}^{2}} \leq 21 \quad\left[\operatorname{sign}_{w}=-, \text { no theory error }\right], \\
& \kappa_{c}\left|s_{c}\right| \sqrt{1+0.78 \kappa_{c} c_{c}+0.047 \kappa_{c}^{2} c_{c}^{2}} \leq 25 \quad\left[\operatorname{sign}_{w}=-, \text { with short-distance theory error }\right], \\
& \kappa_{c}\left|s_{c}\right| \sqrt{1-0.31 \kappa_{c} c_{c}+0.024 \kappa_{c}^{2} c_{c}^{2}} \leq 13 \quad\left[\operatorname{sign}_{w}=+, \text { no theory error }\right],
\end{aligned}
$$




$$
\begin{aligned}
& \kappa_{c}\left|s_{c}\right| \sqrt{1-0.35 \kappa_{c} c_{c}+0.011 \kappa_{c}^{2} c_{c}^{2}} \leq 14 \quad\left[\operatorname{sign}_{w}=+, \text { with short-distance theory error }\right], \\
& \kappa_{c}\left|s_{c}\right| \sqrt{1-0.61 \kappa_{c} c_{c}-0.010 \kappa_{c}^{2} c_{c}^{2}} \leq 19 \quad\left[\operatorname{sign}_{w}=+ \text {, with theory error }\right] .
\end{aligned}
$$

Due to the large theory uncertainties there is no $68.27 \%$ CL interval for the case $\operatorname{sign}_{w}=-$ when the full theory uncertainties are included.

Other hadronic EDMs give, in principle, complementary bounds. For instance, the contribution to the mercury EDM is given by [19]

$$
\frac{d_{\mathrm{Hg}}}{e}=-1.8 \times 10^{-4}\left(4_{-2}^{+8}\right)\left(\tilde{d}_{u}-\tilde{d}_{d}\right) .
$$

Using the current upper experimental 95\% CL bound $\left|d_{\mathrm{Hg}}\right|<3.1 \times 10^{-29} e \mathrm{~cm}[44]$ we compute the allowed $68.27 \%$ CL intervals from the two-parameter $\chi^{2}$. The presently large hadronic uncertainty in eq. (4.18) does not constrain the parameter space at the $68.27 \%$ CL. We thus include only the theory uncertainties associated to short-distance dynamics in our bounds. For the bottom case we find the allowed $68.27 \%$ CL regions to be:

$$
\begin{aligned}
& \kappa_{b}\left|s_{b}\right| \sqrt{1-0.012 \kappa_{b} c_{b}+0.000035 \kappa_{b}^{2} c_{b}^{2}} \leq 8.9 \quad \text { [no theory error], } \\
& \kappa_{b}\left|s_{b}\right| \sqrt{1-0.014 \kappa_{b} c_{b}+0.000037 \kappa_{b}^{2} c_{b}^{2}} \leq 9.6 \quad \text { [with short-distance theory error] }
\end{aligned}
$$

For the charm case we find

$$
\begin{aligned}
\kappa_{c}\left|s_{c}\right| \sqrt{1+0.0087 \kappa_{c} c_{c}+0.000019 \kappa_{c}^{2} c_{c}^{2}} \leq 44 \quad \text { [no theory error] } \\
\kappa_{c}\left|s_{c}\right| \sqrt{1+0.010 \kappa_{c} c_{c}-0.000056 \kappa_{c}^{2} c_{c}^{2}} \leq 48 \quad \text { [with short-distance theory error] }
\end{aligned}
$$

We see that even if we neglect the present theory uncertainties the constraints from mercury EDM cannot compete with the ones from the neutron EDM.

It is instructive to compare the constraints obtained from hadronic EDMs to the constraints from the bound on the electron EDM, obtained recently by the ACME collaboration. The contribution of a modified bottom Yukawa to the electron EDM can be easily obtained by the substitutions $Q_{q} \rightarrow Q_{e}$ and $m_{q} \rightarrow m_{e}$ in eq. (2.4), and similarly for a modified charm Yukawa. Using the ACME result, $\left|d_{e}\right|<1.1 \times 10^{-29} e \mathrm{~cm} \quad(90 \% \mathrm{CL})$ [45], we compute the corresponding allowed intervals. The electron EDM depends solely on the combination $\kappa_{q} \sin \phi_{q}$, which is thus constrained by a one-parameter $\chi^{2}$ function to be $\kappa_{b}\left|s_{b}\right| \leq 0.32$ and $\kappa_{c}\left|s_{c}\right| \leq 0.82$ at the $68.26 \%$ CL for the bottom- and charm-quark case, respectively. To compare with the neutron and mercury EDM allowed two-parameter $68.26 \%$ CL intervals we also list the corresponding ones for the electron EDM:

$$
\begin{array}{ll}
\kappa_{b}\left|s_{b}\right| \leq 0.48 & \text { [no theory error] } \\
\kappa_{c}\left|s_{c}\right| \leq 1.2 & \text { [no theory error] }
\end{array}
$$

Since for the electron EDM there is no hadronic input, the theory uncertainties originate solely from higher electromagnetic corrections and are small. ${ }^{2}$ We see that currently the

\footnotetext{
${ }^{2}$ Our bound on the bottom Yukawa seems consistent with the one recently obtained in ref. [46].
} 
bound from the electron EDM is stronger than the one from the neutron EDM. However, both experimental progress and the anticipated lattice calculations will strengthen the bounds from neutron and other hadronic EDMs. The combination of leptonic and hadronic EDMs can also be used as a strategy to disentangle effect of having multiple CP-violating Yukawas.

We illustrate the results of this section for the bottom- and charm-quark cases in figures 8 and 9, respectively. We show in colour the allowed $68.26 \%$ CL regions of the twoparameter space for different EDMs. In the plots on the left we take the two parameters to be $\kappa_{b(c)} \sin \phi_{b(c)}$ and $\kappa_{b(c)} \cos \phi_{b(c)}$; in the plots on the right we choose the parameters to be $\kappa_{b(c)}$ and $\phi_{b(c)}$. In the upper plots we have included no theory uncertainties; in the lower ones we folded in the theory uncertainties associated to short-distance dynamics.

\section{Conclusions}

We presented the complete two-loop QCD anomalous-dimension matrix for the mixing of CP-odd scalar and tensor operators in an EFT valid below the electroweak-symmetry breaking scale. We used the results to perform a next-to-leading-logarithmic RG analysis of the Wilson coefficients from the weak scale to the hadronic scale of $2 \mathrm{GeV}$, calculating also the requisite finite matching corrections at the heavy-flavor thresholds.

We applied our calculation to a new-physics scenario where new, flavor-conserving, CPviolating phases appear in the Higgs Yukawa couplings to the bottom or charm quark. We calculated the initial conditions at the weak scale up to NLO, and solved the RG equations to compute the induced coefficients of the $\mathrm{CP}$-violating electric and chromoelectric dipole operators and the Weinberg operator in the three-flavor EFT at the hadronic scale $2 \mathrm{GeV}$.

We find large shifts, as well as a large residual scale and scheme dependence of the dipole Wilson coefficients at next-to-leading-logarithmic order. We interpret this as a hint of a slowly converging perturbation series.

The dipole and Weinberg operators contribute to the electric dipole moment of the neutron and mercury. Assuming a Peccei-Quinn-type solution to the strong CP problem we can then derive constraints on the modified Yukawa couplings from the experimental bound on the neutron EDM. These constraints are currently not as strong as those derived from measurements of the electron EDM, but will play an important role in future global fits to modified Higgs Yukawas. This is true in particular in light of the progress expected in lattice calculations of the hadronic matrix elements, and future improvements regarding the experimental bounds on various hadronic EDMs.

\section{Acknowledgments}

We thank Marco Ciuchini, Martin Gorbahn, Mikolai Misiak, and Michael Ramsey-Musolf for useful discussions, and the JHEP referee for pointing out the inconsistency of our treatment of $\gamma_{5}$ in the previous versions of this paper. This work was performed in part at Aspen Center for Physics, which is supported by National Science Foundation grant PHY-1607611. E.S. is supported by the Fermi Fellowship at the Enrico Fermi Institute 


\section{Bottom Yukawa modification}
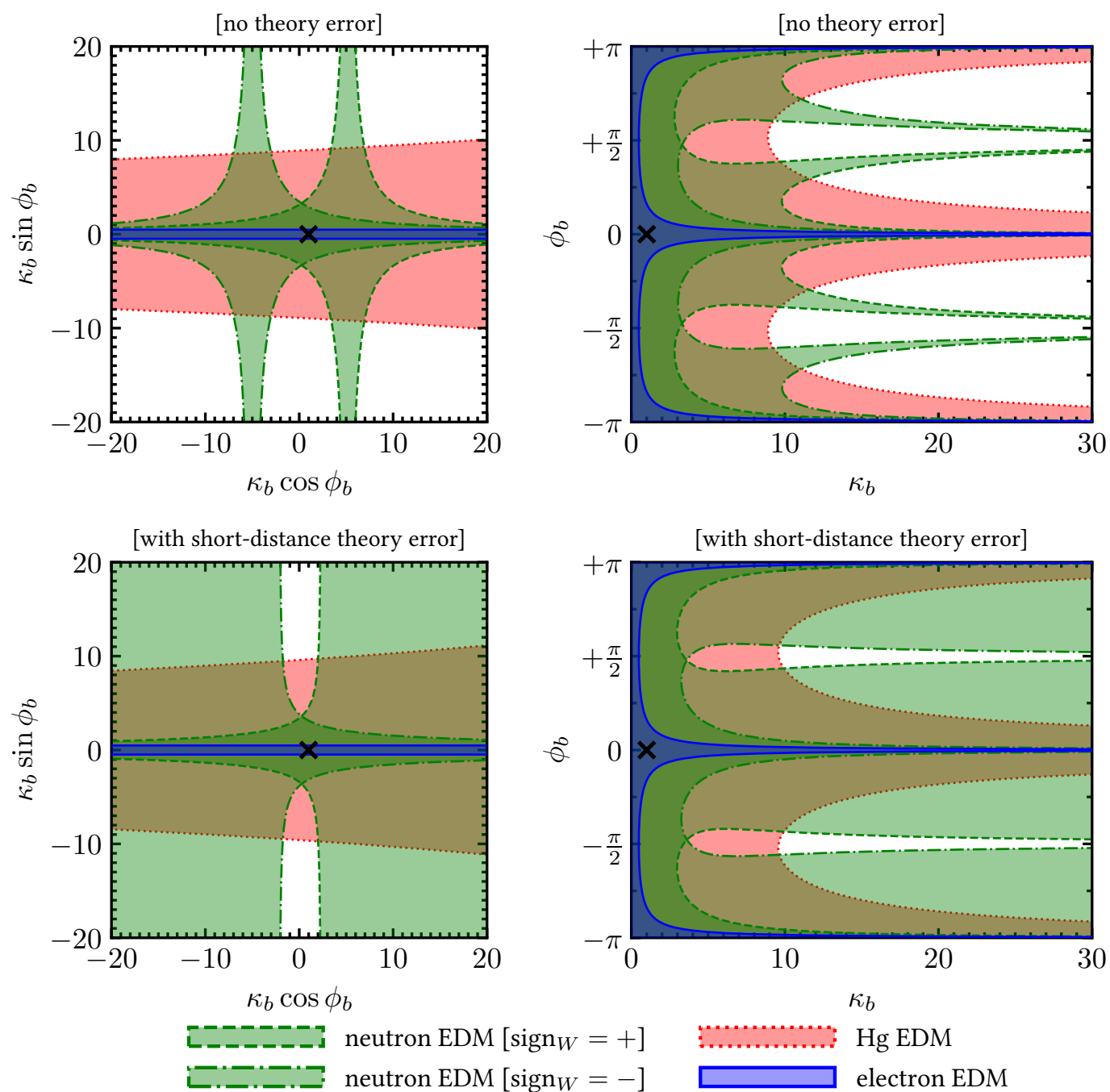

Figure 8. EDM constraints on anomalous CP violating bottom-quark Yukawas. Coloured are the allowed $68.26 \%$ CL regions for the two-parameter space (left: $\kappa_{b} \sin \phi_{b}$ and $\kappa_{b} \cos \phi_{b}$, right: $\kappa_{b}$ and $\left.\phi_{b}\right)$. In green, the allowed region from the neutron EDM for the case of negative (dashed-dotted line) and positive (dashed line) sign for the Weinberg-operator contribution. In red (dotted line) the allowed region from the mercury EDM, and in blue (solid line) the one from the electron EDM.

and by the U.S. Department of Energy, Office of Science, Office of Theoretical Research in High Energy Physics under Award No. DE-SC0009924 and by the Swiss National Science Foundation under contract 200021-178999. J.B. acknowledges support in part by DoE grants DE-SC0020047 and DE-SC0011784.

\section{A Unphysical operators}

Unphysical operators enter our calculation in two different ways. They are needed in order to project all off-shell Greens functions, and they arise as counterterms in intermediate 


\section{Charm Yukawa modification}
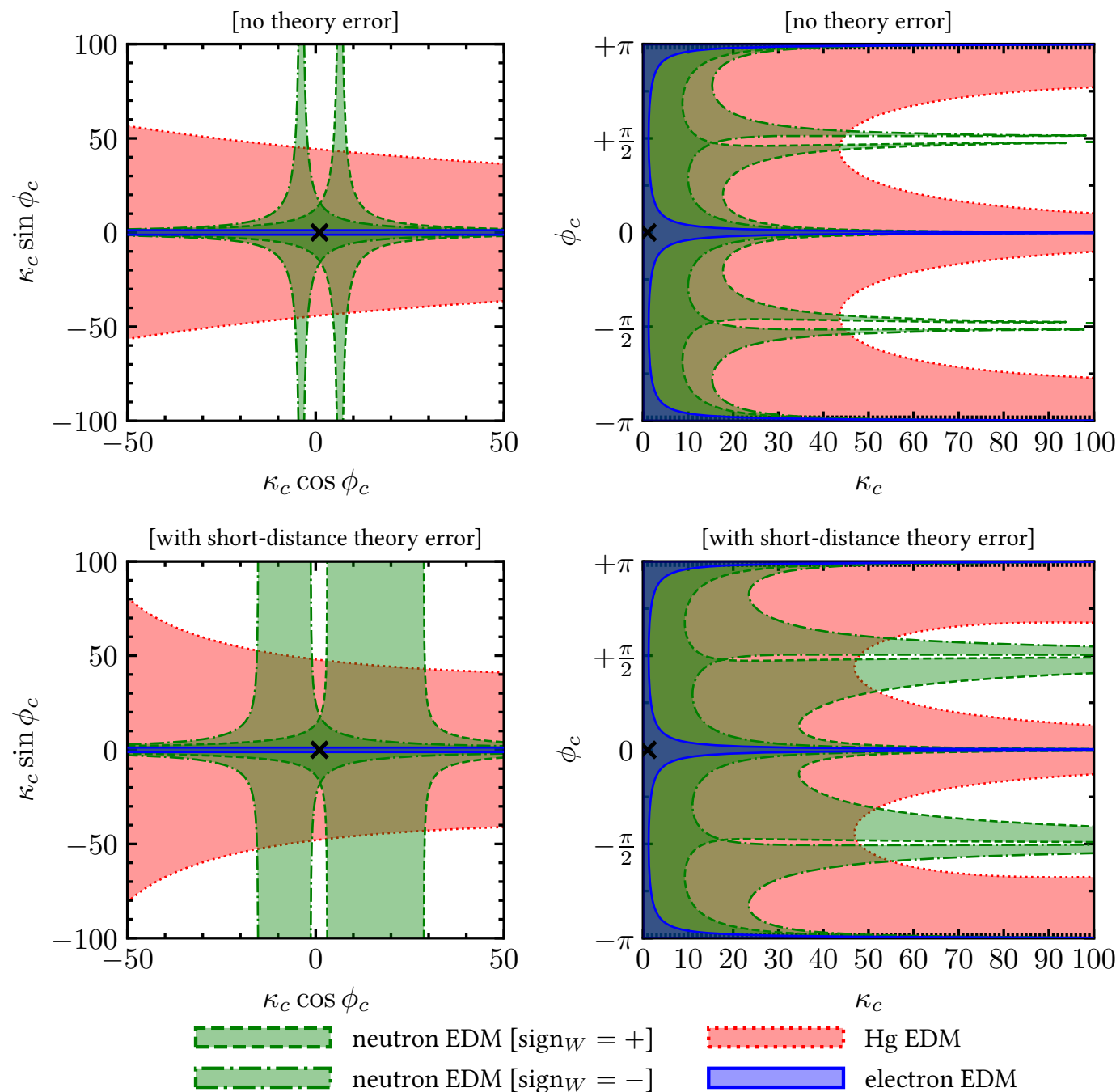

Figure 9. The same as figure 8, but for the case of anomalous CP violating charm-quark Yukawas.

steps of the calculation. They are called "unphysical" because they vanish either via the equations of motion (e.o.m.) of the quark fields for onshell external states, or via algebraic relations that are valid in $d=4$, but not in $d \neq 4$.

\section{A.1 E.o.m.-vanishing operators}

These operators have matrix-elements that vanish via the e.o.m. of the quark field. The following two gauge-invariant ones enter our computation at the two-loop level:

$$
\begin{aligned}
& N_{1}^{q}=\frac{m_{q}}{2 g_{s}^{2}} \bar{q}\left[\overleftarrow{\not D} \overleftarrow{D D} i \gamma_{5}+i \gamma_{5} \not D \not D\right] q \\
& N_{2}^{q}=\frac{m_{q}}{2 g_{s}^{2}} \bar{q}\left[\overleftarrow{D D} \overleftarrow{D^{\sigma}} \gamma^{\mu} \gamma^{\nu} \gamma^{\rho}-\gamma^{\mu} \gamma^{\nu} \gamma^{\rho} D^{\sigma} \not D\right] q \epsilon_{\mu \nu \rho \sigma}
\end{aligned}
$$


Additionally, the following operators, which are not gauge-invariant, are also required in intermediate steps to determine all counterterms and project the off-shell amplitudes

$$
\begin{aligned}
& N_{\gamma 1}^{q}=-\frac{i m_{q} e Q_{q}}{2 g_{s}^{2}} \bar{q}\left[\overleftarrow{\not D} A i \gamma_{5}-i \gamma_{5} A \not D\right] q \\
& N_{\gamma 2}^{q}=-\frac{i m_{q} e Q_{q}}{2 g_{s}^{2}} \bar{q}\left[\overleftarrow{I D} A^{\sigma} \gamma^{\mu} \gamma^{\nu} \gamma^{\rho}+\gamma^{\mu} \gamma^{\nu} \gamma^{\rho} A^{\sigma} \not D\right] q \epsilon_{\mu \nu \rho \sigma}
\end{aligned}
$$

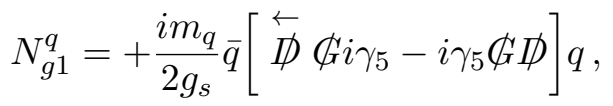

$$
\begin{aligned}
& N_{g 2}^{q}=+\frac{i m_{q}}{2 g_{s}} \bar{q}\left[\overleftarrow{\not D} G^{\sigma} \gamma^{\mu} \gamma^{\nu} \gamma^{\rho}+\gamma^{\mu} \gamma^{\nu} \gamma^{\rho} G^{\sigma} \not D\right] q \epsilon_{\mu \nu \rho \sigma} \\
& N_{g g g}=\frac{1}{g_{s}} f^{a b c} G^{\mu, a} \widetilde{G}_{\mu \nu}^{b}\left(D_{\rho} G^{\rho \nu}\right)^{c} .
\end{aligned}
$$

The covariant derivative acting on quarks is defined as

$$
D_{\mu} \equiv \partial_{\mu}-i g_{s} T^{a} G_{\mu}^{a}+i e Q_{q} A_{\mu}
$$

with $Q_{q}$ the quark electrical charge. Accordingly, the gluon field-strength tensor is

$$
G_{\mu \nu}^{a} \equiv \partial_{\mu} G_{\nu}^{a}-\partial_{\nu} G_{\mu}^{a}+g_{s} f^{a b c} G_{\mu}^{b} G_{\nu}^{c}
$$

The covariant derivative acting on color octets is given by

$$
D_{\mu}^{a b} \equiv \partial_{\mu} \delta^{a b}-g_{s} f^{a b c} G_{\mu}^{c}
$$

\section{A.2 Evanescent operators}

Next we list the evanescent operators that enter our computation at one and two-loop order. The leading-order anomalous dimension does not depend on their definition, but the next-to-leading-order one does.

In the $q-q-A$ sector we require the operator

$$
E_{\gamma}^{q}=\frac{e Q_{q}}{4} \frac{m_{q}}{g_{s}^{2}} \bar{q}\left\{\sigma^{\mu \nu}, i \gamma_{5}\right\} q F_{\mu \nu}-Q_{3}^{q}
$$

Analogously, in the $q-q-G$ sector we require the operator

$$
E_{g}^{q}=-\frac{1}{4} \frac{m_{q}}{g_{s}} \bar{q}\left\{\sigma^{\mu \nu}, i \gamma_{5}\right\} T^{a} q G_{\mu \nu}^{a}-Q_{4}^{q} .
$$

Notice that in eqs. (A.6) and (A.7), as well as in those that follow, we have defined the $\gamma$-algebra structure in terms of anticommutators with $\gamma_{5}$, i.e., $\left\{\Gamma, i \gamma_{5}\right\}$. This ensures that the operators are self-conjugate not just in $d=4$ dimensions but also in $d=4-2 \epsilon$ dimensions. 
The evanescent operators required in the $q-q^{\prime}$ sector read

$$
\begin{aligned}
& E_{1}^{q q^{\prime}}=\frac{1}{2}\left(\bar{q} \gamma_{[\mu} \gamma_{\nu]} q\right)\left(\bar{q}^{\prime}\left\{\gamma^{[\mu} \gamma^{\nu]}, i \gamma_{5}\right\} q^{\prime}\right)+O_{3}^{q q^{\prime}} \\
& E_{2}^{q q^{\prime}}=\frac{1}{2}\left(\bar{q} \gamma_{[\mu} \gamma_{\nu]} T^{a} q\right)\left(\bar{q}^{\prime}\left\{\gamma^{[\mu} \gamma^{\nu]}, i \gamma_{5}\right\} T^{a} q^{\prime}\right)+O_{4}^{q q^{\prime}}, \\
& E_{3}^{q q^{\prime}}=\left[\left(\bar{q} \gamma_{[\mu} \gamma_{\nu]} \gamma^{[\rho} \gamma^{\sigma]} q\right)\left(\bar{q}^{\prime} \gamma^{[\mu} \gamma^{\nu]} \gamma^{[\tau} \gamma^{v]} q^{\prime}\right)+\left(\bar{q} \gamma^{[\rho} \gamma^{\sigma]} \gamma_{[\mu} \gamma_{\nu]} q\right)\left(\bar{q}^{\prime} \gamma^{[\tau} \gamma^{v]} \gamma^{[\mu} \gamma^{\nu]} q^{\prime}\right)\right] \epsilon_{\rho \sigma \tau v} \\
& -48\left(O_{1}^{q q^{\prime}}+O_{1}^{q^{\prime} q}\right)+16 O_{3}^{q q^{\prime}}, \\
& E_{4}^{q q^{\prime}}=\frac{1}{2}\left[\left(\bar{q} \gamma_{[\mu} \gamma_{\nu]} \gamma^{[\rho} \gamma^{\sigma]} T^{a} q\right)\left(\bar{q}^{\prime} \gamma^{[\mu} \gamma^{\nu]} \gamma^{[\tau} \gamma^{v]} T^{a} q^{\prime}\right)\right. \\
& \left.+\left(\bar{q} \gamma^{[\rho} \gamma^{\sigma]} \gamma_{[\mu} \gamma_{\nu]} T^{a} q\right)\left(\bar{q}^{\prime} \gamma^{[\tau} \gamma^{v]} \gamma^{[\mu} \gamma^{\nu]} T^{a} q^{\prime}\right)\right] \epsilon_{\rho \sigma \tau v} \\
& -48\left(O_{2}^{q q^{\prime}}+O_{2}^{q^{\prime} q}\right)+16 O_{4}^{q q^{\prime}}, \\
& E_{5}^{q q^{\prime}}=\frac{1}{2}\left(\bar{q} \gamma_{[\mu} \gamma_{\nu} \gamma_{\rho} \gamma_{\sigma]} q\right)\left(\bar{q}^{\prime}\left\{\gamma^{[\mu} \gamma^{\nu} \gamma^{\rho} \gamma^{\sigma]}, i \gamma_{5}\right\} q^{\prime}\right)-24 O_{1}^{q^{\prime} q} \\
& E_{6}^{q q^{\prime}}=\frac{1}{2}\left(\bar{q} \gamma_{[\mu} \gamma_{\nu} \gamma_{\rho} \gamma_{\sigma]} T^{a} q\right)\left(\bar{q}^{\prime}\left\{\gamma^{[\mu} \gamma^{\nu} \gamma^{\rho} \gamma^{\sigma]}, i \gamma_{5}\right\} T^{a} q^{\prime}\right)-24 O_{2}^{q^{\prime} q}, \\
& E_{7}^{q q^{\prime}}=\frac{1}{2}\left(\bar{q} \gamma_{[\mu} \gamma_{\nu} \gamma_{\rho} \gamma_{\sigma} \gamma_{\tau} \gamma_{v]} q\right)\left(\bar{q}^{\prime}\left\{\gamma^{[\mu} \gamma^{\nu} \gamma^{\rho} \gamma^{\sigma} \gamma^{\tau} \gamma^{v]}, i \gamma_{5}\right\} q^{\prime}\right) \\
& \left.E_{8}^{q q^{\prime}}=\frac{1}{2}\left(\bar{q} \gamma_{[\mu} \gamma_{\nu} \gamma_{\rho} \gamma_{\sigma} \gamma_{\tau} \gamma_{v]} T^{a} q\right)\left(\bar{q}^{\prime} \gamma^{[\mu} \gamma^{\nu} \gamma^{\rho} \gamma^{\sigma} \gamma^{\tau} \gamma^{v]}, i \gamma_{5}\right\} T^{a} q^{\prime}\right) \\
& E_{9}^{q q^{\prime}}=\frac{1}{2}\left[\left(\bar{q} \gamma_{[\mu} \gamma_{\nu} \gamma_{\rho} \gamma_{\sigma]} \gamma^{[\tau} \gamma^{v]} q\right)\left(\bar{q}^{\prime} \gamma^{[\mu} \gamma^{\nu} \gamma^{\rho} \gamma^{\sigma]} \gamma^{[\zeta} \gamma^{\xi]} q^{\prime}\right)\right. \\
& \left.+\left(\bar{q} \gamma^{[\tau} \gamma^{v]} \gamma_{[\mu} \gamma_{\nu} \gamma_{\rho} \gamma_{\sigma]} q\right)\left(\bar{q}^{\prime} \gamma^{[\zeta} \gamma^{\xi]} \gamma^{[\mu} \gamma^{\nu} \gamma^{\rho} \gamma^{\sigma]} q^{\prime}\right)\right] \epsilon_{\tau v \zeta \xi}+48 O_{3}^{q q^{\prime}}, \\
& E_{10}^{q q^{\prime}}=\frac{1}{2}\left[\left(\bar{q} \gamma_{[\mu} \gamma_{\nu} \gamma_{\rho} \gamma_{\sigma]} \gamma^{[\tau} \gamma^{v]} T^{a} q\right)\left(\bar{q}^{\prime} \gamma^{[\mu} \gamma^{\nu} \gamma^{\rho} \gamma^{\sigma]} \gamma^{[\zeta} \gamma^{\xi]} T^{a} q^{\prime}\right)\right. \\
& \left.+\left(\bar{q} \gamma^{[\tau} \gamma^{v]} \gamma_{[\mu} \gamma_{\nu} \gamma_{\rho} \gamma_{\sigma]} T^{a} q\right)\left(\bar{q}^{\prime} \gamma^{[\zeta} \gamma^{\xi]} \gamma^{[\mu} \gamma^{\nu} \gamma^{\rho} \gamma^{\sigma]} T^{a} q^{\prime}\right)\right] \epsilon_{\tau v \zeta \xi}+48 O_{4}^{q q^{\prime}}
\end{aligned}
$$

The square brackets denote antisymmetrisation normalized as

$$
\gamma_{\left[\mu_{1}, \ldots, \mu_{n}\right]} \equiv \frac{1}{n !} \sum_{\sigma}(-1)^{\sigma} \gamma_{\mu_{\sigma(1)}} \ldots \gamma_{\mu_{\sigma(n)}} .
$$

In the $q-q$ sector they read

$$
\begin{aligned}
& E_{1}^{q}=\left(\bar{q} T^{a} q\right)\left(\bar{q} i \gamma_{5} T^{a} q\right)+\left(\frac{1}{4}+\frac{1}{2 n_{c}}\right) O_{1}^{q}+\frac{1}{16} O_{2}^{q}, \\
& E_{2}^{q}=\frac{1}{2}\left(\bar{q} \sigma^{\mu \nu} T^{a} q\right)\left(\bar{q} \sigma^{\rho \sigma} T^{a} q\right) \epsilon_{\mu \nu \rho \sigma}+3 O_{1}^{q}-\left(\frac{1}{4}-\frac{1}{2 n_{c}}\right) O_{2}^{q}, \\
& E_{3}^{q}=\frac{1}{2}\left(\bar{q} \gamma_{[\mu} \gamma_{\nu]} q\right)\left(\bar{q}\left\{\gamma^{[\mu} \gamma^{\nu]}, i \gamma_{5}\right\} q\right)+O_{2}^{q},
\end{aligned}
$$




$$
\begin{aligned}
& E_{4}^{q}=\frac{1}{2}\left(\bar{q} \gamma_{[\mu} \gamma_{\nu]} T^{a} q\right)\left(\bar{q}\left\{\gamma^{[\mu} \gamma^{\nu]}, i \gamma_{5}\right\} T^{a} q\right)-3 O_{1}^{q}+\left(\frac{1}{4}-\frac{1}{2 n_{c}}\right) O_{2}^{q} \\
& E_{5}^{q}=\frac{1}{2}\left[\left(\bar{q} \gamma_{[\mu} \gamma_{\nu]} \gamma^{[\rho} \gamma^{\sigma]} q\right)\left(\bar{q} \gamma^{[\mu} \gamma^{\nu]} \gamma^{[\tau} \gamma^{v]} q\right)+\left(\bar{q} \gamma^{[\rho} \gamma^{\sigma]} \gamma_{[\mu} \gamma_{\nu]} q\right)\left(\bar{q} \gamma^{[\tau} \gamma^{v]} \gamma^{[\mu} \gamma^{\nu]} q\right)\right] \epsilon_{\rho \sigma \tau v} \\
& -96 O_{1}^{q}+16 O_{2}^{q}, \\
& E_{6}^{q}=\frac{1}{2}\left[\left(\bar{q} \gamma_{[\mu} \gamma_{\nu]} \gamma^{[\rho} \gamma^{\sigma]} T^{a} q\right)\left(\bar{q} \gamma^{[\mu} \gamma^{\nu]} \gamma^{[\tau} \gamma^{\nu]} T^{a} q\right)\right. \\
& \left.+\left(\bar{q} \gamma^{[\rho} \gamma^{\sigma]} \gamma_{[\mu} \gamma_{\nu]} T^{a} q\right)\left(\bar{q} \gamma^{[\tau} \gamma^{v]} \gamma^{[\mu} \gamma^{\nu]} T^{a} q\right)\right] \epsilon_{\rho \sigma \tau v} \\
& -24\left(1-\frac{2}{n_{c}}\right) O_{1}^{q}+2\left(5-\frac{4}{n_{c}}\right) O_{2}^{q} \\
& E_{7}^{q}=\frac{1}{2}\left(\bar{q} \gamma_{[\mu} \gamma_{\nu} \gamma_{\rho} \gamma_{\sigma]} q\right)\left(\bar{q}\left\{\gamma^{[\mu} \gamma^{\nu} \gamma^{\rho} \gamma^{\sigma]}, i \gamma_{5}\right\} q\right)-24 O_{1}^{q} \\
& E_{8}^{q}=\frac{1}{2}\left(\bar{q} \gamma_{[\mu} \gamma_{\nu} \gamma_{\rho} \gamma_{\sigma]} T^{a} q\right)\left(\bar{q}\left\{\gamma^{[\mu} \gamma^{\nu} \gamma^{\rho} \gamma^{\sigma]}, i \gamma_{5}\right\} T^{a} q\right)+6\left(1+\frac{2}{n_{c}}\right) O_{1}^{q}+\frac{3}{2} O_{2}^{q}, \\
& E_{9}^{q}=\frac{1}{2}\left(\bar{q} \gamma_{[\mu} \gamma_{\nu} \gamma_{\rho} \gamma_{\sigma} \gamma_{\tau} \gamma_{v]} q\right)\left(\bar{q}\left\{\gamma^{[\mu} \gamma^{\nu} \gamma^{\rho} \gamma^{\sigma} \gamma^{\tau} \gamma^{v]}, i \gamma_{5}\right\} q\right), \\
& E_{10}^{q}=\frac{1}{2}\left(\bar{q} \gamma_{[\mu} \gamma_{\nu} \gamma_{\rho} \gamma_{\sigma} \gamma_{\tau} \gamma_{v]} T^{a} q\right)\left(\bar{q}\left\{\gamma^{[\mu} \gamma^{\nu} \gamma^{\rho} \gamma^{\sigma} \gamma^{\tau} \gamma^{v]}, i \gamma_{5}\right\} T^{a} q\right) \\
& E_{11}^{q}=\frac{1}{2}\left[\left(\bar{q} \gamma_{[\mu} \gamma_{\nu} \gamma_{\rho} \gamma_{\sigma]} \gamma^{[\tau} \gamma^{v]} q\right)\left(\bar{q} \gamma^{[\mu} \gamma^{\nu} \gamma^{\rho} \gamma^{\sigma]} \gamma^{[\zeta} \gamma^{\xi]} q\right)\right. \\
& \left.+\left(\bar{q} \gamma^{[\tau} \gamma^{v]} \gamma_{[\mu} \gamma_{\nu} \gamma_{\rho} \gamma_{\sigma]} q\right)\left(\bar{q} \gamma^{[\zeta} \gamma^{\xi]} \gamma^{[\mu} \gamma^{\nu} \gamma^{\rho} \gamma^{\sigma]} q\right)\right] \epsilon_{\tau v \zeta \xi} \\
& +48 O_{2}^{q} \\
& E_{12}^{q}=\frac{1}{2}\left[\left(\bar{q} \gamma_{[\mu} \gamma_{\nu} \gamma_{\rho} \gamma_{\sigma]} \gamma^{[\tau} \gamma^{v]} T^{a} q\right)\left(\bar{q} \gamma^{[\mu} \gamma^{\nu} \gamma^{\rho} \gamma^{\sigma]} \gamma^{[\zeta} \gamma^{\xi]} T^{a} q\right)\right. \\
& \left.+\left(\bar{q} \gamma^{[\tau} \gamma^{v]} \gamma_{[\mu} \gamma_{\nu} \gamma_{\rho} \gamma_{\sigma]} T^{a} q\right)\left(\bar{q} \gamma^{[\zeta} \gamma^{\xi]} \gamma^{[\mu} \gamma^{\nu} \gamma^{\rho} \gamma^{\sigma]} T^{a} q\right)\right] \epsilon_{\tau v \zeta \xi} \\
& -144 O_{1}^{q}+12\left(1-\frac{2}{n_{c}}\right) O_{2}^{q} .
\end{aligned}
$$

In simplifying the color algebra, we use the following standard relation for the generators of $\mathrm{SU}\left(n_{c}\right)$ :

$$
\sum_{a} T_{i j}^{a} T_{k l}^{a}=\frac{1}{2} \delta_{i l} \delta_{j k}-\frac{1}{2 n_{c}} \delta_{i j} \delta_{k l} .
$$

Consequently, Fierz relations on the Lorentz structures, valid in $d=4$, need to be applied on the operators with $T^{a}$ 's to show that they are evanescent. 


\section{A.3 Operators related to the infrared rearrangement}

The last class of unphysical operators arises because our infrared rearrangement breaks gauge invariance in intermediate steps of the calculation. At the renormalizable level this method generates one gauge-variant operator corresponding to a gluon-mass term, i.e.,

$$
\mathscr{L} \supset \frac{1}{2} Z_{\mathrm{IRA}} m_{\mathrm{IRA}}^{2} G_{\mu}^{a} G^{\mu, a} .
$$

The "gluon mass", $m_{\mathrm{IRA}}$, is completely artificial and drops out of all physical results, and $Z_{\mathrm{IRA}}$ is an additional renormalisation constant [32]. At the non-renormalizable level the one-loop insertions of the dimension-five and dimension-six operators can also induce gauge-invariant operators that are relics of the infrared rearrangement. For our calculation, the only relevant ones are the following three

$$
\begin{aligned}
& P^{q}=m_{q} \frac{m_{\mathrm{IRA}}^{2}}{g_{s}^{2}} \bar{q} i \gamma_{5} q, \\
& P_{\gamma}^{q}=\frac{e Q_{q} m_{\mathrm{IRA}}^{2}}{2 g_{s}^{2}} \bar{q}\left\{A, i \gamma_{5}\right\} q, \\
& P_{g}^{q}=-\frac{m_{\mathrm{IRA}}^{2}}{2 g_{s}} \bar{q}\left\{\phi_{r}, i \gamma_{5}\right\} q .
\end{aligned}
$$

\section{B Renormalisation constants}

To obtain the two-loop anomalous dimension of the physical sector we need certain oneloop renormalisation constants involving unphysical operators. We collect them in this appendix. We use the following standard notation for their expansion in $\alpha_{s}$ and $\epsilon$

$$
Z_{x \rightarrow y}=\sum_{k} \sum_{l=1}^{k} \frac{\alpha_{s}^{k}}{(4 \pi)^{k} \epsilon^{l}} Z_{x \rightarrow y}^{(k, l)} .
$$

In $\overline{\mathrm{MS}}$, the mixing of evanescent operators into physical also includes finite terms [47], thus in this case the $\epsilon$ expansion starts with $l=0$. The subscripts $x$ and $y$ symbolize sets of Wilson coefficients, for which we use the following notation and standard ordering:

$$
\begin{aligned}
& q=\left\{C_{1}^{q}, C_{2}^{q}, C_{3}^{q}, C_{4}^{q}\right\}, \\
& q q^{\prime}=\left\{C_{1}^{q q^{\prime}}, C_{2}^{q q^{\prime}}, C_{1}^{q^{\prime} q}, C_{2}^{q^{\prime} q}, C_{3}^{q q^{\prime}}, C_{4}^{q q^{\prime}}\right\}, \\
& E^{q}=\left\{C_{E_{1}^{q}}, C_{E_{2}^{q}}, C_{E_{4}^{q}}, C_{E_{6}^{q}}\right\}, \\
& E^{q q^{\prime}}=\left\{C_{E_{1}^{q q^{\prime}}}, C_{E_{2}^{q q^{\prime}}}, C_{E_{1}^{q^{\prime} q}}, C_{E_{2}^{q^{\prime} q}}, C_{E_{3}^{q q^{\prime}}}, C_{E_{4}^{q q^{\prime}}}\right\}, \\
& E_{\gamma g}^{q}=\left\{C_{E_{\gamma}^{q}}, C_{E_{g}^{q}}\right\}, \\
& P^{q}=\left\{C_{P^{q}}, C_{P_{\gamma}^{q}}, C_{P_{g}^{q}}\right\}, \\
& N^{q}=\left\{C_{N_{1}^{q}}, C_{N_{2}^{q}}, C_{N_{\gamma 1}^{q}}, C_{N_{\gamma 2}^{q}}, C_{N_{g 1}^{q}} C_{N_{g 2}^{q}}\right\} .
\end{aligned}
$$


The first necessary input is the mixing of the physical operators into all the evanescent operators that are generated at one-loop. Using the same subscript notation as above, the renormalisation constants read

$$
\begin{aligned}
Z_{q \rightarrow E^{q}}^{(1,1)} & =\left(\begin{array}{cccc}
0 & 0 & 1 & 0 \\
0 & -8 & 0 & -\frac{1}{2} \\
0 & 0 & 0 & 0 \\
0 & 0 & 0 & 0
\end{array}\right), \quad Z_{q q^{\prime} \rightarrow E^{q q^{\prime}}}^{(1,1)}=\left(\begin{array}{cccccc}
0 & 1 & 0 & 0 & 0 & 0 \\
\frac{2}{9} & \frac{5}{12} & 0 & 0 & 0 & 0 \\
0 & 0 & 0 & 1 & 0 & 0 \\
0 & 0 & \frac{2}{9} & \frac{5}{12} & 0 & 0 \\
0 & 0 & 0 & 0 & 0 & -\frac{1}{2} \\
0 & 0 & 0 & 0 & -\frac{1}{9} & -\frac{5}{24}
\end{array}\right) \\
Z_{q \rightarrow E_{\gamma g}^{q}}^{(1,1)} & =\left(\begin{array}{cc}
2 & 2 \\
24 & 24 \\
0 & 0 \\
-\frac{16}{3} & \frac{11}{3}
\end{array}\right) .
\end{aligned}
$$

The remaining mixings of physical operators into evanescent operators are all zero at oneloop. Furthermore, the finite part of the mixing of evanescent into physical operators is subtracted by finite counterterms [47]. They read

$$
\begin{aligned}
& Z_{E^{q} \rightarrow q}^{(1,0)}=\left(\begin{array}{cccc}
-\frac{5}{8} & -\frac{3}{32} & 0 & 0 \\
-\frac{9}{2} & \frac{1}{8} & 0 & 0 \\
\frac{169}{36} & -\frac{11}{48} & -\frac{16}{3} & \frac{2}{3} \\
22 & -\frac{235}{18} & -\frac{128}{3} & \frac{16}{3}
\end{array}\right), \quad Z_{E^{q q^{\prime}} \rightarrow q q^{\prime}}^{(1,0)}=\left(\begin{array}{cccccc}
0 & 4 & 0 & 0 & 0 & 0 \\
\frac{8}{9} & \frac{5}{3} & 0 & 0 & 0 & 0 \\
0 & 0 & 0 & 4 & 0 & 0 \\
0 & 0 & \frac{8}{9} & \frac{5}{3} & 0 & 0 \\
0 & 0 & 0 & 0 & 0 & -8 \\
0 & 0 & 0 & 0 & -\frac{16}{9} & -\frac{10}{3}
\end{array}\right), \\
& Z_{E_{\gamma g}^{q} \rightarrow q}^{(1,0)}=\left(\begin{array}{cccc}
0 & 0 & 0 & 0 \\
0 & 0 & -\frac{8}{9} & \frac{11}{18}
\end{array}\right) .
\end{aligned}
$$

The remaining finite mixings of evanescent into physical operators are zero.

Furthermore, we need the mixing constants of the physical operators into the operators arising from infrared rearrangement; they are found to be

$$
Z_{q \rightarrow P^{q}}^{(1,1)}=\left(\begin{array}{ccc}
-10 & -2 & -2 \\
24 & -24 & -24 \\
0 & 0 & 0 \\
-16 & 0 & 0
\end{array}\right), \quad Z_{q q^{\prime} \rightarrow P^{q}}^{(1,1)}=\left(\begin{array}{ccc}
0 & 0 & 0 \\
0 & 0 & 0 \\
-12 \frac{m_{q^{\prime}}}{m_{q}} & 0 & 0 \\
0 & 0 & 0 \\
0 & 0 & 0 \\
0 & 0 & 0
\end{array}\right)
$$


All other mixing constants of physical into the IRA operators are zero. Special care must be taken to obtain the mixings like $Z_{q q^{\prime} \rightarrow P^{\prime}}^{(1,1)}$. Apart from the obvious $q \leftrightarrow q^{\prime}$ interchange also the ordering of the operators in the collective blocks is relevant. For example

$$
Z_{q q^{\prime} \rightarrow P^{q^{\prime}}}^{(1,1)}=\left(\begin{array}{rrrr}
-12 \frac{m_{q}}{m_{q^{\prime}}} & 0 & 0 \\
0 & 0 & 0 \\
0 & 0 & 0 \\
0 & 0 & 0 \\
0 & 0 & 0 \\
0 & 0 & 0
\end{array}\right)
$$

Finally, we need the mixing constants of the physical operators into the e.o.m.-vanishing operators. They are uniquely fixed by the $q \rightarrow q, q \rightarrow q \gamma$, and $q \rightarrow q g$ Greens functions and we have verified that their values are consistent with the renormalisation of the $q g \rightarrow q \gamma$ and $q g \rightarrow q g$ Greens functions. We find

$$
Z_{q \rightarrow N^{q}}^{(1,1)}=\left(\begin{array}{cccccc}
0 & 0 & 0 & 0 & 0 & 0 \\
0 & 0 & 0 & 0 & 0 & 0 \\
0 & 0 & 0 & 0 & 0 & 0 \\
-\frac{8}{3} & -\frac{2}{9} & 0 & 0 & 0 & \frac{3}{8}
\end{array}\right) .
$$

The two-loop anomalous dimension matrix is given in terms of the one- and two-loop renormalisation constants by

$$
\gamma^{(1)}=4 Z^{(2,1)}-2 Z^{(1,1)} Z^{(1,0)}-2 Z^{(1,0)} Z^{(1,1)}+2 \beta_{0} Z^{(1,0)} .
$$

The quadratic poles of the two-loop diagrams are fixed by the poles of the one-loop diagrams via

$$
Z^{(2,2)}=\frac{1}{2} Z^{(1,1)} Z^{(1,1)}-\frac{1}{2} \beta_{0} Z^{(1,1)},
$$

where $\beta_{0}=\frac{11}{3} n_{c}-\frac{2}{3} N_{f}$. As a check of our calculation, we computed these poles directly and verified that they satisfy eq. (B.8).

In our calculation we needed various field and mass renormalisation constants up to two-loop level, and we have calculated them explicitly. Writing the expansion

$$
Z_{r}=\sum_{k} \sum_{l=1}^{k} \frac{\alpha_{s}^{k}}{(4 \pi)^{k} \epsilon^{l}} Z_{r}^{(k, l)}
$$

with $r=q, m, g_{s}, G, u, m_{\mathrm{IRA}}$ denoting the quark field, quark mass, strong coupling, gluon 
field, ghost field, and artificial gluon mass renormalisation, respectively, we find

$$
\begin{aligned}
& Z_{q}^{(1,1)}=-\frac{\left(n_{c}^{2}-1\right)}{2 n_{c}} \xi_{g}, \\
& Z_{m}^{(1,1)}=-\frac{3\left(n_{c}^{2}-1\right)}{2 n_{c}}, \\
& Z_{g_{s}}^{(1,1)}=-\frac{11}{6} n_{c}+\frac{1}{3} N_{f}, \\
& Z_{\mathrm{IRA}}^{(1,1)}=-\frac{n_{c}}{4}\left(1+3 \xi_{g}\right)-2 N_{f}, \\
& Z_{G}^{(1,1)}=\left(\frac{13}{6}-\frac{1}{2} \xi_{g}\right) n_{c}-\frac{2}{3} N_{f}, \\
& Z_{u}^{(1,1)}=\frac{n_{c}\left(3-\xi_{g}\right)}{4}, \\
& { }_{q}^{(2,2)}=\frac{\left(n_{c}^{2}-1\right)\left[n_{c}^{2}\left(2 \xi_{g}+3\right)-\xi_{g}\right]}{8 n_{c}^{2}} \xi_{g}, \\
& Z_{m}^{(2,2)}=-\frac{\left(n_{c}^{2}-1\right)\left(9-31 n_{c}^{2}+4 n_{c} N_{f}\right)}{8 n_{c}^{2}}, \\
& Z_{g_{s}}^{(2,2)}=\frac{121}{24} n_{c}^{2}-\frac{11}{6} n_{c} N_{f}+\frac{1}{6} N_{f}^{2}, \\
& Z_{G}^{(2,2)}=\left(-\frac{13}{8}-\frac{17}{24} \xi_{g}+\frac{1}{4} \xi_{g}^{2}\right) n_{c}^{2}+\left(\frac{1}{2}+\frac{1}{3} \xi_{g}\right) n_{c} N_{f}, \\
& Z_{q}^{(2,1)}=-\frac{\left(n_{c}^{2}-1\right)\left[n_{c}^{2}\left(\xi_{g}^{2}+8 \xi_{g}+22\right)-4 n_{c} N_{f}+3\right]}{16 n_{c}^{2}}, \\
& Z_{m}^{(2,1)}=\frac{\left(n_{c}^{2}-1\right)\left(9-203 n_{c}^{2}+20 n_{c} N_{f}\right)}{48 n_{c}^{2}}, \\
& Z_{g_{s}}^{(2,1)}=-\frac{17}{6} n_{c}^{2}-\frac{1}{4 n_{c}} N_{f}+\frac{13}{12} n_{c} N_{f}, \\
& Z_{G}^{(2,1)}=\left(\frac{59}{16}-\frac{11}{16} \xi_{g}-\frac{1}{8} \xi_{g}^{2}\right) n_{c}^{2}-\left(\frac{7}{4} n_{c}-\frac{1}{2 n_{c}}\right) N_{f},
\end{aligned}
$$

with $\xi_{g}$ the gauge fixing parameter in generalized $R_{\xi}$ gauge. Our renormalisation constants agree with the results in the literature [48] if one bears in mind that the original papers contain some typographical errors.

\section{The one-loop anomalous dimensions}

Using the same notation as in the previous section, we decompose the anomalous dimension matrix of the physical sector in subblocks. The anomalous dimension matrices $\gamma_{x \rightarrow y}$ admit 
a perturbative expansion in the strong coupling constant,

$$
\gamma=\frac{\alpha_{s}}{4 \pi} \gamma^{(0)}+\left(\frac{\alpha_{s}}{4 \pi}\right)^{2} \gamma^{(1)}+\ldots
$$

The one-loop anomalous dimension matrix is given in terms of the renormalisation constants by

$$
\gamma^{(0)}=2 Z^{(1,1)}
$$

The explicit results read

$$
\begin{aligned}
& \gamma_{q \rightarrow q}^{(0)}=\left(\begin{array}{cccc}
-10 & -\frac{1}{6} & 4 & 4 \\
40 & \frac{34}{3} & -112 & -16 \\
0 & 0 & -\frac{34}{3}+\frac{4}{3} N_{f} & 0 \\
0 & 0 & \frac{32}{3} & -\frac{38}{3}+\frac{4}{3} N_{f}
\end{array}\right) \\
& \gamma_{q q^{\prime} \rightarrow q}^{(0)}=\left(\begin{array}{cccc}
0 & 0 & 0 & 0 \\
0 & 0 & 0 & 0 \\
0 & 0 & 0 & 0 \\
0 & 0 & 0 & 0 \\
0 & 0 & -48 \frac{Q_{q^{\prime}}}{Q_{q}} \frac{m_{q^{\prime}}}{m_{q}} & 0 \\
0 & 0 & 0 & -8 \frac{m_{q^{\prime}}}{m_{q}}
\end{array}\right) \\
& \gamma_{q q^{\prime} \rightarrow q q^{\prime}}^{(0)}=\left(\begin{array}{cccccc}
-16 & 0 & 0 & 0 & 0 & -2 \\
0 & 2 & 0 & 0 & -\frac{4}{9} & -\frac{5}{6} \\
0 & 0 & -16 & 0 & 0 & -2 \\
0 & 0 & 0 & 2 & -\frac{4}{9} & -\frac{5}{6} \\
0 & -48 & 0 & -48 & \frac{16}{3} & 0 \\
-\frac{32}{3} & -20 & -\frac{32}{3} & -20 & 0 & -\frac{38}{3}
\end{array}\right), \\
& \gamma_{W \rightarrow q}^{(0)}=\left(\begin{array}{llll}
0 & 0 & 0 & 6
\end{array}\right) \text {, } \\
& \gamma_{W \rightarrow W}^{(0)}=-8+\frac{8}{3} N_{f} .
\end{aligned}
$$

All other physical subblocks are zero at one-loop. Our one-loop results for the physical sector agree with the results in the literature $[29,49,50]$, after accounting for the different normalisation of the operators and the different conventions in the covariant derivative.

\section{Change of renormalisation scheme}

The (three-loop) mixing among the dipole operators $O_{3}^{q}$ and $O_{4}^{q}$ has already been calculated in the literature [31] (see ref. [30] for the earlier two-loop result for the CP-even dipole operators). In this section we show that our results are consistent with these calculations. 
There are three main differences between our calculation and the earlier ones: (i) the definition of the physical operators differs in $d$ space-time dimensions, (ii) the calculation in ref. [31] has effectively been performed in the NDR scheme whereas ours in the "Larin" scheme, and (iii) a different normalisation of the operators with factors of quark masses and the strong coupling has been chosen. Here we show that there is a unique change of the renormalisation scheme that transforms our result into that of refs. [30, 31].

To address (i), we perform a redefinition of our basis of physical operators as follows: ${ }^{3}$

$$
\begin{aligned}
& O_{3}^{\prime q} \equiv O_{3}^{q}+E_{\gamma}^{q}=\frac{e Q_{q}}{4} \frac{m_{q}}{g_{s}^{2}} \bar{q}\left\{\sigma^{\mu \nu}, i \gamma_{5}\right\} q F_{\mu \nu}, \\
& O_{4}^{\prime q} \equiv O_{4}^{q}+E_{g}^{q}=-\frac{1}{4} \frac{m_{q}}{g_{s}} \bar{q}\left\{\sigma^{\mu \nu}, i \gamma_{5}\right\} T^{a} q G_{\mu \nu}^{a} .
\end{aligned}
$$

All other physical operators are unchanged, i.e. $Q_{i}^{\prime} \equiv Q_{i}$. The resulting operators correspond, in the NDR scheme, to the operators used in refs. [30, 31], up to a normalisation (see below).

To address (ii), we mimick the results obtained in the NDR scheme by redefining the evanescent operator $E_{g}^{q}$ to be:

$$
E_{g}^{\prime q} \equiv E_{g}^{q}+\frac{4}{47} \epsilon O_{3}^{q}-\frac{19}{94} \epsilon O_{4}^{q}
$$

with all other operators unchanged. The coefficients in front of the physical operators are uniquely determined by the requirement to reproduce the anomalous dimensions of the dipole operators in the literature.

Finally, to address (iii) we take care of the different overall normalisation of the operators as follows. If we write the shifted renormalisation constants as $Z^{\prime}=\rho Z$, where

$$
\rho=1+\frac{\alpha_{s}}{4 \pi \epsilon} \rho^{(1,1)}+\left(\frac{\alpha_{s}}{4 \pi \epsilon}\right)^{2}\left(\rho^{(2,2)}+\epsilon \rho^{(2,1)}\right),
$$

then the shifted ADMs are given by

$$
\gamma^{\prime(0)}=\gamma^{(0)}+2 \rho^{(1,1)}, \quad \gamma^{\prime(1)}=\gamma^{(1)}+4 \rho^{(2,1)} .
$$

Combining this shift with the change of physical and evanescent operators specified above (see, e.g., ref. [51] for the general expressions), we can reproduce both the one- and twoloop mixing of the dipole operators in refs. [30,31]. The mixing of four-fermion into dipole operators, on the other hand, cannot be calculated in the NDR scheme. For completeness,

\footnotetext{
${ }^{3}$ The lattice results used in our numerics have been converted to the NDR- $\overline{\mathrm{MS}}$ scheme at the one-loop level [40]. If, in the future, the two-loop conversion of the lattice results becomes available, one should perform the change of scheme discussed in this appendix for our analysis.
} 
we provide the full physical two-loop anomalous dimension matrix in the primed basis:

$$
\begin{aligned}
& \gamma_{q q^{\prime} \rightarrow q}^{(1)}=\left(\begin{array}{cccc}
0 & 0 & \frac{32}{27} \frac{m_{q^{\prime}}}{m_{q}} & -\frac{640}{47} \frac{m_{q^{\prime}}}{m_{q}} \\
0 & 0 & \frac{40}{141} \frac{m_{q^{\prime}}}{m_{q}}-16 \frac{Q_{q^{\prime}}}{Q_{q}} \frac{m_{q^{\prime}}}{m_{q}} & -\frac{800}{141} \frac{m_{q^{\prime}}}{m_{q}} \\
0 & 0 & \frac{32}{27} \frac{m_{q^{\prime}}}{m_{q}} & -\frac{264}{47} \frac{m_{q^{\prime}}}{m_{q}} \\
0 & 0 & \frac{40}{141} \frac{m_{q^{\prime}}}{m_{q}}-\frac{16}{3} \frac{Q_{q^{\prime}}}{Q_{q}} \frac{m_{q^{\prime}}}{m_{q}} & -\frac{110}{47} \frac{m_{q^{\prime}}}{m_{q}} \\
0 & 0 & -448 \frac{Q_{q^{\prime}}}{Q_{q}} \frac{m_{q^{\prime}}}{m_{q}} & 0 \\
0 & 0 & \frac{2976}{47} \frac{m_{q^{\prime}}}{m_{q}} & -\frac{20932}{141} \frac{m_{q^{\prime}}}{m_{q}}
\end{array}\right) \\
& \gamma_{q \rightarrow q}^{(1)}=\left(\begin{array}{cccc}
65-6 N_{f} & \frac{N_{f}}{54}-\frac{19}{12} & \frac{26896}{1269} & \frac{81211}{1269} \\
60-\frac{40 N_{f}}{9} & \frac{403}{3}-\frac{226 N_{f}}{27} & -\frac{339776}{423} & -\frac{65780}{423} \\
0 & 0 & \frac{460 N_{f}}{27}+\frac{100}{9} & 0 \\
0 & 0 & \frac{368}{3}-\frac{224 N_{f}}{27}-\frac{380 N_{f}}{9}+\frac{623}{27}
\end{array}\right) .
\end{aligned}
$$

The matrix $\gamma_{q q^{\prime} \rightarrow q q^{\prime}}^{(1)}$ does not change by our redefinition of operators.

\section{E Expanding the renormalisation group}

To gain a better understanding of our results, and as an additional check of our calculation, we expand the full solution of the RG equations about the bottom-quark threshold (the procedure for the charm quark is analogous).

Keeping only the leading nonvanishing terms and including the QED contribution, eq. (2.4), we find for the electric dipole

$$
\begin{aligned}
d_{q}= & \sqrt{2} G_{F} \frac{Q_{q} e m_{q}}{g_{s}^{2}} C_{3}^{q}\left(m_{b}\right) \\
\simeq & \sqrt{2} G_{F} \frac{Q_{q} e m_{q}}{g_{s}^{2}}\left\{-12 Q_{b}^{2} \frac{\alpha \alpha_{s}\left(m_{b}\right)}{(4 \pi)^{2}} \kappa_{b} \sin \phi_{b} x_{b}\left(\log ^{2} x_{b}+\frac{\pi^{2}}{3}\right)\right. \\
& +\left(\frac{\alpha_{s}\left(m_{b}\right)}{4 \pi}\right)^{3}\left(C_{1}^{q q^{\prime}(0)}\left(M_{h}\right) \frac{\gamma_{1, q q^{\prime} \rightarrow 4, q q^{\prime}}^{(0)} \gamma_{4, q q^{\prime} \rightarrow 4, q}^{(0)} \gamma_{4, q \rightarrow 3, q}^{(0)}}{48} \log ^{3} x_{b}\right. \\
& +C_{1}^{q q^{\prime}(0)}\left(M_{h}\right) \frac{\gamma_{1, q q^{\prime} \rightarrow 4, q q^{\prime}}^{(0)} \gamma_{4, q q^{\prime} \rightarrow 3, q}^{(1)}+\gamma_{1, q q^{\prime} \rightarrow 4, q}^{(1)} \gamma_{4, q \rightarrow 3, q}^{(0)}+\gamma_{1, q q^{\prime} \rightarrow 3, q q^{\prime}}^{(1)} \gamma_{3, q q^{\prime} \rightarrow 3, q}^{(0)}}{8} \log ^{2} x_{b} \\
& +C_{4}^{q q^{\prime}(1)}\left(M_{h}\right) \frac{\left.\left.\gamma_{4, q q^{\prime} \rightarrow 4, q}^{(0)} \gamma_{4, q \rightarrow 3, q}^{(0)} \log ^{2} x_{b}\right)\right\}}{8} \\
= & \sqrt{2} G_{F} Q_{q} e m_{q} \kappa_{b} \sin \phi_{b} x_{b} \\
& \times\left\{-12 Q_{b}^{2} \frac{\alpha}{(4 \pi)^{3}}\left(\log ^{2} x_{b}+\frac{\pi^{2}}{3}\right)-\frac{32}{9} \frac{\alpha_{s}^{2}\left(m_{b}\right)}{(4 \pi)^{4}} \log ^{3} x_{b}+\frac{32}{3} \frac{\alpha_{s}^{2}\left(m_{b}\right)}{(4 \pi)^{4}} \log ^{2} x_{b}\right\},
\end{aligned}
$$


where the superscripts "(0)" and "(1)" denote the tree-level and one-loop contributions to the initial conditions of the Wilson coefficients at the weak scale (we omit here the small logarithmic contributions $\left.\propto \log \left(\mu_{\mathrm{ew}}^{2} / M_{h}^{2}\right)\right)$. Using $\alpha_{s}\left(m_{b}\right) \sim 0.22$, the ratio of QED to LL to NLL is roughly $1:-9:-4$. We see (as observed already in ref. [3]) that the contribution of the photonic Barr-Zee diagram is negligible. We also see that the NLL correction is quite large, about half the size of the LL contribution.

The leading terms in the expansion of the solution for the RG equations for the chromoelectric dipole, on the other hand, should exactly reproduce the logarithmic parts of the result in eq. (2.4) (the constant term is of next-to-next-to-leading logarithmic order and can only be reproduced, within EFT, by a three-loop calculation). Indeed, we find

$$
\begin{gathered}
\tilde{d}_{q}=-\sqrt{2} G_{F} \frac{m_{q}}{g_{s}^{2}} C_{4}^{q}\left(m_{b}\right) \\
\simeq-\sqrt{2} G_{F} \frac{m_{q}}{g_{s}^{2}}\left(\frac{\alpha_{s}\left(m_{b}\right)}{4 \pi}\right)^{2}\left\{C_{1}^{q q^{\prime}(0)}\left(M_{h}\right) \frac{\gamma_{1, q q^{\prime} \rightarrow 4, q q^{\prime}}^{(0)} \gamma_{4, q q^{\prime} \rightarrow 4, q}^{(0)}}{8} \log ^{2} x_{b}\right. \\
-C_{1}^{q q^{\prime}(0)}\left(M_{h}\right) \frac{\gamma_{1, q q^{\prime} \rightarrow 4, q}^{(1)}}{2} \log x_{b} \\
\left.-C_{4}^{q q^{\prime}(1)}\left(M_{h}\right) \frac{\gamma_{4, q q^{\prime} \rightarrow 4, q}^{(0)}}{2} \log x_{b}\right\} \\
=2 \frac{\alpha_{s}\left(m_{b}\right)}{(4 \pi)^{3}} \sqrt{2} G_{F} m_{q} \kappa_{b} \sin \phi_{b} x_{b} \log ^{2} x_{b}+0 .
\end{gathered}
$$

As expected, the leading contribution to the LL reproduces the quadratic logarithm in eq. (2.4), while leading contribution to the NLL result vanishes.

This means, in turn, that the NLL corrections to $\tilde{d}_{q}$ start at relative order $\alpha_{s} /(4 \pi)$, with large anomalous-dimension prefactors of $\left(\gamma_{1, q q^{\prime} \rightarrow 1, q q^{\prime}}^{(0)} \gamma_{1, q q^{\prime} \rightarrow 4, q}^{(1)}+\gamma_{1, q q^{\prime} \rightarrow 4, q q^{\prime}}^{(0)} \gamma_{4, q q^{\prime} \rightarrow 4, q}^{(1)}\right) / 8=$ 47 and $\left(\gamma_{1, q q^{\prime} \rightarrow 4, q}^{(1)} \gamma_{4, q \rightarrow 4, q}^{(0)}+\gamma_{1, q q^{\prime} \rightarrow 4, q q^{\prime}}^{(1)} \gamma_{4, q q^{\prime} \rightarrow 4, q}^{(0)}\right) / 8=404 / 9$, multiplied by the LO initial condition -1 , and $\left(\gamma_{4, q q^{\prime} \rightarrow 4, q}^{(0)} \gamma_{4, q \rightarrow 4, q}^{(0)}+\gamma_{4, q q^{\prime} \rightarrow 4, q q^{\prime}}^{(0)} \gamma_{4, q q^{\prime} \rightarrow 4, q}^{(0)}\right) / 8=56 / 3$, multiplied by the NLO initial condition $3 / 2$. Therefore, these constitute sizeable relative corrections, with ratio $1:-0.6$ between the LL and the NLL contributions. Needless to say that in our numerics we use the full solution of the RG equations, where all large logarithms are resummed to leading and next-to-leading order.

For completeness we give the also expansion of the Dicus result [52]:

$$
\begin{aligned}
w & =-\frac{\sqrt{2} G_{F}}{g_{s}} C_{w}\left(m_{b}\right) \\
& \simeq-\frac{\sqrt{2} G_{F}}{g_{s}}\left(\frac{\alpha_{s}\left(m_{b}\right)}{4 \pi}\right)^{2}\left\{-\frac{1}{2} C_{1}^{q(0)}\left(M_{h}\right) \frac{\gamma_{1, q \rightarrow 4, q}^{(0)}}{2} \log x_{b}\right\} \\
& =-\sqrt{2} G_{F} \frac{g_{s}\left(m_{b}\right) \alpha_{s}\left(m_{b}\right)}{(4 \pi)^{3}} \kappa_{b}^{2} \sin \phi_{b} \cos \phi_{b} x_{b} \log x_{b} .
\end{aligned}
$$

This reproduces the logarithmic term in eq. (2.4). 
Open Access. This article is distributed under the terms of the Creative Commons Attribution License (CC-BY 4.0), which permits any use, distribution and reproduction in any medium, provided the original author(s) and source are credited.

\section{References}

[1] D.E. Morrissey and M.J. Ramsey-Musolf, Electroweak baryogenesis, New J. Phys. 14 (2012) 125003 [arXiv: 1206 .2942] [INSPIRE].

[2] S.J. Huber, M. Pospelov and A. Ritz, Electric dipole moment constraints on minimal electroweak baryogenesis, Phys. Rev. D 75 (2007) 036006 [hep-ph/0610003] [InSPIRE].

[3] J. Brod, U. Haisch and J. Zupan, Constraints on CP-violating Higgs couplings to the third generation, JHEP 11 (2013) 180 [arXiv:1310.1385] [INSPIRE].

[4] R. Harnik, A. Martin, T. Okui, R. Primulando and F. Yu, Measuring CP-violation in $h \rightarrow \tau^{+} \tau^{-}$at Colliders, Phys. Rev. D 88 (2013) 076009 [arXiv:1308.1094] [InSPIRE].

[5] W. Altmannshofer, J. Brod and M. Schmaltz, Experimental constraints on the coupling of the Higgs boson to electrons, JHEP 05 (2015) 125 [arXiv: 1503.04830] [INSPIRE].

[6] Y.T. Chien, V. Cirigliano, W. Dekens, J. de Vries and E. Mereghetti, Direct and indirect constraints on CP-violating Higgs-quark and Higgs-gluon interactions, JHEP 02 (2016) 011 [arXiv: 1510.00725] [INSPIRE].

[7] V. Cirigliano, W. Dekens, J. de Vries and E. Mereghetti, Is there room for CP-violation in the top-Higgs sector?, Phys. Rev. D 94 (2016) 016002 [arXiv: 1603.03049] [INSPIRE].

[8] V. Cirigliano, W. Dekens, J. de Vries and E. Mereghetti, Constraining the top-Higgs sector of the Standard Model Effective Field Theory, Phys. Rev. D 94 (2016) 034031 [arXiv: 1605.04311] [INSPIRE].

[9] J. Brod and D. Skodras, Electric dipole moment constraints on CP-violating light-quark Yukawas, JHEP 01 (2019) 233 [arXiv:1811.05480] [INSPIRE].

[10] T. Bhattacharya, V. Cirigliano, R. Gupta, H.-W. Lin and B. Yoon, Neutron Electric Dipole Moment and Tensor Charges from Lattice QCD, Phys. Rev. Lett. 115 (2015) 212002 [arXiv: 1506. 04196] [INSPIRE].

[11] PNDME collaboration, Iso-vector and Iso-scalar Tensor Charges of the Nucleon from Lattice QCD, Phys. Rev. D 92 (2015) 094511 [arXiv:1506.06411] [INSPIRE].

[12] T. Bhattacharya, V. Cirigliano, R. Gupta, E. Mereghetti and B. Yoon, Dimension-5 CP-odd operators: QCD mixing and renormalization, Phys. Rev. D 92 (2015) 114026 [arXiv: 1502.07325] [INSPIRE].

[13] T. Bhattacharya, V. Cirigliano, R. Gupta and B. Yoon, Quark Chromoelectric Dipole Moment Contribution to the Neutron Electric Dipole Moment, PoS LATTICE 2016 (2016) 225 [arXiv: 1612.08438] [INSPIRE].

[14] F. Feruglio, The Chiral approach to the electroweak interactions, Int. J. Mod. Phys. A 8 (1993) 4937 [hep-ph/9301281] [INSPIRE].

[15] G. Buchalla, O. Catà and C. Krause, Complete Electroweak Chiral Lagrangian with a Light Higgs at NLO, Nucl. Phys. B 880 (2014) 552 [Erratum ibid. 913 (2016) 475] [arXiv: 1307.5017] [INSPIRE]. 
[16] R. Alonso, E.E. Jenkins, A.V. Manohar and M. Trott, Renormalization Group Evolution of the Standard Model Dimension Six Operators III: Gauge Coupling Dependence and Phenomenology, JHEP 04 (2014) 159 [arXiv:1312.2014] [INSPIRE].

[17] J. Engel, M.J. Ramsey-Musolf and U. van Kolck, Electric Dipole Moments of Nucleons, Nuclei, and Atoms: The Standard Model and Beyond, Prog. Part. Nucl. Phys. 71 (2013) 21 [arXiv: 1303.2371] [INSPIRE].

[18] S. Weinberg, Larger Higgs Exchange Terms in the Neutron Electric Dipole Moment, Phys. Rev. Lett. 63 (1989) 2333 [inSPIRE].

[19] M. Pospelov and A. Ritz, Electric dipole moments as probes of new physics, Annals Phys. 318 (2005) 119 [hep-ph/0504231] [INSPIRE].

[20] S.M. Barr and A. Zee, Electric Dipole Moment of the Electron and of the Neutron, Phys. Rev. Lett. 65 (1990) 21 [Erratum ibid. 65 (1990) 2920] [INSPIRE].

[21] E. Braaten, C.-S. Li and T.-C. Yuan, The Evolution of Weinberg's Gluonic CP Violation Operator, Phys. Rev. Lett. 64 (1990) 1709 [InSPIRE].

[22] E. Braaten, C.-S. Li and T.-C. Yuan, The Gluon Color-Electric Dipole Moment and Its Anomalous Dimension, Phys. Rev. D 42 (1990) 276 [inSPIRE].

[23] Particle Data collaboration, Review of Particle Physics, Prog. Theor. Exp. Phys. 2020 (2020) 083C01 [INSPIRE].

[24] G. Buchalla, A.J. Buras and M.E. Lautenbacher, Weak decays beyond leading logarithms, Rev. Mod. Phys. 68 (1996) 1125 [hep-ph/9512380] [InSPIRE].

[25] J.A.M. Vermaseren, New features of FORM, math-ph/0010025 [INSPIRE].

[26] A.I. Davydychev and J.B. Tausk, Two loop selfenergy diagrams with different masses and the momentum expansion, Nucl. Phys. B 397 (1993) 123 [INSPIRE].

[27] C. Bobeth, M. Misiak and J. Urban, Photonic penguins at two loops and $m_{t}$ dependence of $B R\left[B \rightarrow X_{s} l^{+} l^{-}\right]$, Nucl. Phys. B 574 (2000) 291 [hep-ph/9910220] [InSPIRE].

[28] P. Nogueira, Automatic Feynman graph generation, J. Comput. Phys. 105 (1993) 279 [INSPIRE].

[29] J. Hisano, K. Tsumura and M.J.S. Yang, QCD Corrections to Neutron Electric Dipole Moment from Dimension-six Four-Quark Operators, Phys. Lett. B 713 (2012) 473 [arXiv: 1205.2212] [INSPIRE].

[30] M. Misiak and M. Münz, Two loop mixing of dimension five flavor changing operators, Phys. Lett. B 344 (1995) 308 [hep-ph/9409454] [INSPIRE].

[31] M. Gorbahn, U. Haisch and M. Misiak, Three-loop mixing of dipole operators, Phys. Rev. Lett. 95 (2005) 102004 [hep-ph/0504194] [INSPIRE].

[32] K.G. Chetyrkin, M. Misiak and M. Münz, $\beta$-functions and anomalous dimensions up to three loops, Nucl. Phys. B 518 (1998) 473 [hep-ph/9711266] [INSPIRE].

[33] J.C. Collins, Renormalization, in Cambridge Monographs on Mathematical Physics 26, Cambridge University Press, Cambridge U.K. (1986).

[34] S.A. Larin, The Renormalization of the axial anomaly in dimensional regularization, Phys. Lett. B 303 (1993) 113 [hep-ph/9302240] [INSPIRE]. 
[35] J. de Vries, G. Falcioni, F. Herzog and B. Ruijl, Two- and three-loop anomalous dimensions of Weinberg's dimension-six CP-odd gluonic operator, Phys. Rev. D 102 (2020) 016010 [arXiv: 1907.04923] [INSPIRE].

[36] K.G. Chetyrkin, B.A. Kniehl and M. Steinhauser, Decoupling relations to $\mathcal{O}\left(\alpha_{s}^{3}\right)$ and their connection to low-energy theorems, Nucl. Phys. B 510 (1998) 61 [hep-ph/9708255] [INSPIRE].

[37] A.J. Buras and P.H. Weisz, QCD Nonleading Corrections to Weak Decays in Dimensional Regularization and 't Hooft-Veltman Schemes, Nucl. Phys. B 333 (1990) 66 [InSPIRE].

[38] S. Herrlich and U. Nierste, Evanescent operators, scheme dependences and double insertions, Nucl. Phys. B 455 (1995) 39 [hep-ph/9412375] [INSPIRE].

[39] J. Brod, J. Cornell, D. Skodras and E. Stamou, Global Constraints on Yukawa Operators in the Standard Model Effective Theory, to appear (2021).

[40] JLQCD collaboration, Nucleon charges with dynamical overlap fermions, Phys. Rev. D 98 (2018) 054516 [arXiv: 1805.10507] [INSPIRE].

[41] Flavour Lattice Averaging Group, FLAG Review 2019: Flavour Lattice Averaging Group (FLAG), Eur. Phys. J. C 80 (2020) 113 [arXiv: 1902.08191] [INSPIRE].

[42] R. Gupta, B. Yoon, T. Bhattacharya, V. Cirigliano, Y.-C. Jang and H.-W. Lin, Flavor diagonal tensor charges of the nucleon from $(2+1+1)$-flavor lattice QCD, Phys. Rev. D 98 (2018) 091501 [arXiv: 1808. 07597] [INSPIRE].

[43] C.A. Baker et al., An Improved experimental limit on the electric dipole moment of the neutron, Phys. Rev. Lett. 97 (2006) 131801 [hep-ex/0602020] [INSPIRE].

[44] W.C. Griffith, M.D. Swallows, T.H. Loftus, M.V. Romalis, B.R. Heckel and E.N. Fortson, Improved Limit on the Permanent Electric Dipole Moment of ${ }^{199} \mathrm{Hg}$, Phys. Rev. Lett. 102 (2009) 101601 [arXiv:0901.2328] [INSPIRE].

[45] ACME collaboration, Improved limit on the electric dipole moment of the electron, Nature 562 (2018) 355 [INSPIRE].

[46] D. Egana-Ugrinovic and S. Thomas, Higgs Boson Contributions to the Electron Electric Dipole Moment, arXiv:1810.08631 [INSPIRE].

[47] M.J. Dugan and B. Grinstein, On the vanishing of evanescent operators, Phys. Lett. B 256 (1991) 239 [INSPIRE].

[48] P. Gambino, M. Gorbahn and U. Haisch, Anomalous dimension matrix for radiative and rare semileptonic B decays up to three loops, Nucl. Phys. B 673 (2003) 238 [hep-ph/0306079] [INSPIRE].

[49] A.J. Buras, M. Misiak and J. Urban, Two loop QCD anomalous dimensions of flavor changing four quark operators within and beyond the standard model, Nucl. Phys. B 586 (2000) 397 [hep-ph/0005183] [INSPIRE].

[50] G. Degrassi, E. Franco, S. Marchetti and L. Silvestrini, QCD corrections to the electric dipole moment of the neutron in the MSSM, JHEP 11 (2005) 044 [hep-ph/0510137] [INSPIRE].

[51] J. Brod and M. Gorbahn, $\epsilon_{K}$ at Next-to-Next-to-Leading Order: The Charm-Top-Quark Contribution, Phys. Rev. D $\mathbf{8 2}$ (2010) 094026 [arXiv: 1007.0684] [INSPIRE].

[52] D.A. Dicus, Neutron Electric Dipole Moment From Charged Higgs Exchange, Phys. Rev. D 41 (1990) 999 [INSPIRE]. 